\title{
Modulation of Myosin by Cardiac Myosin Binding Protein-C Peptides Improves Cardiac Contractility in Ex-Vivo Experimental Heart Failure Models
}

\section{Luqia Hou}

Merck \& Co., Inc.

\section{Mohit Kumar}

University of Cincinnati

\section{Priti Anand}

Merck \& Co., Inc.

Yinhong Chen

Merck \& Co., Inc.

\section{Nesrine El-Bizri}

Merck \& Co., Inc.

\section{Chad J Pickens}

Merck \& Co., Inc.

Michael Seganish

Merck \& Co., Inc.

\section{Sakthivel Sadayappan}

University of Cincinnati

Gayathri Swaminath ( $\sim$ gswaminath@gmail.com )

Merck \& Co., Inc.

\section{Research Article}

Keywords: cMyBP-C, myosin, peptide, myocardial infarction, heart failure, HCM and DCM

Posted Date: November 12th, 2021

DOI: https://doi.org/10.21203/rs.3.rs-955920/v1

License: (1) (i) This work is licensed under a Creative Commons Attribution 4.0 International License.

Read Full License

Version of Record: A version of this preprint was published at Scientific Reports on March 14th, 2022. See the published version at https://doi.org/10.1038/s41598-022-08169-1. 


\section{Abstract}

Cardiac myosin binding protein-C (cMyBP-C) is an important regulator of sarcomeric function. Although reduced phosphorylation of $\mathrm{CMyBP-C}$ has been linked to compromised contractility in heart failure patients, direct modulation of CMyBP-C to myosin using small molecules or peptides has not been reported to improve cardiac performance. Here we used previously published cMyBP-C peptides 302A and $302 S$ (surrogates to the regulatory phosphorylation site serine 302) as tool molecules to investigate the role of CMyBP-C in modulating cardiac contraction and relaxation in experimental heart failure (HF) models in vitro. CMyBP-C peptides 302A and 302S were able to increase contractility of papillary muscle fibers isolated from a cMyBP-C phospho-ablation (cMyBP-C ${ }^{A A A}$ ) mouse model. In addition, 302A was able to improve the force redevelopment rate $\left(k_{\mathrm{tr}}\right)$ in papillary muscle fibers from cMyBP-CAAA mice. Consistent with above findings, CMyBP-C peptides 302A and 302S were able to increase the ATPase rates in myofibrils isolated from $\mathrm{MI}$ rats but not from sham rats. Furthermore, in CMyBP-C ${ }^{A A A}$ mouse and myocardial infarction (MI) HF models, both cMyBP-C peptides 302A and 302S were able to improve ATPase hydrolysis rates. These changes were not observed in non-transgenic (NTG) mice or sham rats, indicating the specific effects of these peptides in regulating the reduced or unphosphorylated state of CMyBP-C only under pathological conditions of heart failure. Taken together, these studies demonstrate that modulation of CMyBP-C in a reduced phosphorylation or unphosphorylated state can be a therapeutic approach to improve myosin function, sarcomere contractility and relaxation. Therefore, targeting CMyBP-C can be a differentiated approach to improve overall cardiac performance on top of standard care drugs in HF patients.

\section{Introduction}

Cardiac myosin binding protein-C (cMyBP-C) is a $140-\mathrm{kDa}$-sarcomeric-thick filament protein ${ }^{1,2}$ which localizes in regular intervals at the $\mathrm{C}$ zone of the sarcomere and regulates sarcomere structure and function in the heart ${ }^{3-5}$. The regulation of CMyBP-C comes from three phosphorylation sites at the Mdomain and via interaction of its N-terminal-region with both myosin and actin. CMyBP-C is highly phosphorylated under normal physiological conditions and its phosphorylation levels decrease in diseased states both in preclinical heart failure (HF) models and clinically in patients with hypertrophic cardiomyopathy (HCM), HF or atrial fibrillation. Despite the clear benefits of targeting cMyBP-C, there is no drug on the market to directly modify cMyBP-C properties to improve cardiac function.

Human and mouse cMyBP-C proteins have three phosphorylation sites in the M-domain at the N-terminus of the molecule, all of which are lacking in the skeletal muscle isoform ${ }^{3,6}$. The region of the $\mathrm{N}$-terminus binds to the $\mathrm{S} 2$ segment of myosin, close to the lever-arm domain ${ }^{7-9}$. This interaction can be dynamically regulated by phosphorylation/dephosphorylation of cMyBP-C. After phosphorylation of cMyBP-C by PKA, the thick filaments exhibit a loose structure, which promotes cooperative cross-bridge cycling by relieving cMyBP-C's normal inhibition on myosin and increasing proximity towards the actin on cMyBP-C's own binding to the thin filament, thus contributing to improved cardiac function ${ }^{10}$. On the 
other hand, dephosphorylated CMyBP-C has greater myosin interaction, shortens myosin cross-bridge duration, and directly decreases cardiac contractility ${ }^{11-14}$. Similarly, the catalytic cleavage of the Nterminal region of cMyBP-C during MI reduces the number of phosphorylation sites, leading to depressed cardiac contractility and function ${ }^{15}$.

In previous studies, the necessity and sufficiency of cMyBP-C phosphorylation for regulating normal cardiac function was determined using cardiac-specific transgenic (TG) mice expressing phosphoablated cMyBP-C at Ser-273-Ala/Ser-282-Ala/Ser-302-Ala sites (AAA) or phospho-mimetic cMyBP-C at

Ser-273-Asp/Ser-282-Asp/Ser-302-Asp (DDD) compared to non-transgenic (NTG) control mice ${ }^{16-19}$. Using the same animal model, PKA-mediated phosphorylation of CMyBP-C was shown to contribute to enhancing the Frank-Starling regulatory mechanism of the heart ${ }^{20}$. Moreover, an age-dependent negative regulation of CMyBP-C dephosphorylation was reported in similar models ${ }^{16,21,22}$. To therapeutically target cMyBP-C in order to improve cardiac function and HF, we explored two preclinical models reported in the literature. In the first model, all three serine phosphorylation sites (273, 282 and 302) were mutated to non-phosphorylatable alanines (AAA) ${ }^{17}$; in another model, all three serine phosphorylation sites were mutated to phospho-mimetic aspartic acids (DDD) ${ }^{18}$ to mimic the physiological state of the heart. AAA replacement resulted in depressed cardiac function, and the protein was unable to rescue the CMyBP-C null phenotype ${ }^{23}$, while the TG mouse expressed cMyBP-C wild-type and NTG controls ${ }^{17}$. Conversely, DDD was able to rescue the cMyBP-C null phenotype.

In this study, we applied a different approach to target CMyBP-C by using peptides that were designed based on the sequence of the M-domain of the CMyBP-C protein. These peptides were evaluated in vitro, and 302A (peptide surrogate to phosphorylation site at 302) was able to improve force generation and calcium sensitivity in cardiac muscle fibers ${ }^{24}$. However, the mechanism by which these peptides improve contractile kinetics relative to the phosphorylation status of cMyBP-C has not been fully elucidated. We hypothesized that the 302A peptide would modify the binding properties of cMyBP-C to myosin and improve cardiac performance under pathological conditions. To test this hypothesis, we evaluated the effects of cMyBP-C peptides 302A and 302S in three different in vitro assays: a steady-state ATPase assay, a myofibril ATPase assay, and a papillary muscle assay. Data generated from in vitro assays and pre-clinical heart failure models provides strong evidence that modulating phosphorylation status of cMyBP-C increases ATPase activity and kinetics, which can improve cardiac performance.

\section{Results}

\section{CMyBP-C phosphorylation was reduced in the Rat MI heart failure model}

To test the effect of cMyBP-C phosphorylation status in the MI-HF model, we collected protein samples from the left ventricular (LV) infarct zone of MI rats and a comparable area from sham animals at five different time points post-MI surgery: day 1, day 3, week 1, week 4 and week 8 (Fig. 1, Sup. Fig. 2). CMyBP-C phosphorylation was detected using antibodies specifically targeting serine phosphorylation 
sites at positions 273, 282 and 302, as well as targeting the total cMyBP-C. Alpha-actin was used as a housekeeping protein for normalization on day 1 and day 3 while HPRT was used as a housekeeping protein for normalization at week 1 , week 4 , and week 8 post-MI due to significantly reduced alpha-actin levels after week 1 post-MI. The findings suggest that the expression levels of all three phosphorylated serine residues (p273, p282 and p302) were reduced significantly in early time points (day 1 and day 3 ) followed by a trend in reduction at week 1 post-MI samples, which became significant in myofibril samples isolated from the same time point (Fig. 4). Surprisingly, there was a significant increase in the expression of all three phosphorylation sites at later time points (week 4 and week 8 post-MI samples; Sup. Fig. 2) except for p273 at week 8 , which could be due to possible compensation effect during heart failure progression. We noticed that the expression of total CMyBP-C closely followed the trend of cMyBP$C$ phosphorylation changes (Fig. 1 and Sup. Fig. 2), indicating the main effect of cardiac injury was on total cMyBP-C expression. We also tested the expression of Troponin T and I, which showed a consistent reduction through the time points: day 1, day 3, week 1, week 4 and week 8 (Sup. Fig. 3). Interestingly, reduced expression of the myosin heavy chain (MHC) was delayed and was observed only on day 3 postMI. There was a quick compensation on MHC expression with a dramatic increase at later time points (week 4 and week 8 post-Ml; Sup. Fig. 3).

\section{CMyBP-C phosphorylation was reduced in human HCM and DCM patients}

To test the phosphorylation status of CMyBP-C in human HF, IHC was performed using cardiac tissue sections from normal healthy donors, as well as hypertrophic cardiomyopathy (HCM) and dilated cardiomyopathy (DCM) patients ( $\mathrm{N}=4$ patients per group, Fig. 2). HCM and DCM sample tissues were confirmed by a trained physiologist. IHC was performed using antibodies against p273 and p282, as well as an antibody against total CMyBP-C. Relative expression of p273 was slightly reduced in cardiac tissues from HCM but not DCM patients (Normal 0.72 \pm 0.08 vs HCM 0.48 $\pm 0.06, p=0.06$; DCM 0.78 \pm 0.10 ; $\mathrm{n}=16$; Fig. 2B). On the other hand, relative expression of $\mathrm{p} 282$ was reduced significantly in both HCM and DCM patients (Normal $1.64 \pm 0.16$ vs HCM 0.93 \pm 0.06 , $p<0.005$; Normal $1.64 \pm 0.16$ vs DCM $1.04 \pm 0.11$, $\mathrm{p}<0.01)$. The immunohistochemistry $(\mathrm{IHC})$ data from human patients strongly supported the reduced CMyBP-C phosphorylation in human heart failure.

\section{cMyBP-C peptide 302A did not improve ATPase activity in recombinant steady-state ATPase assay}

Next we decided to test the effect of cMyBP-C peptides (Table I) in a steady-state ATPase assay, which involved myosin, actin and full-length (FL) CMyBP-C or a truncated C0-C2 segment (either unphosphorylated $[-P]$ or phosphorylated $[+P])$. ATPase activity was measured by detecting the $\mathrm{Pi}$ released from ATP hydrolysis. Inhibition of myosin ATPase activity was observed with increasing concentrations of both $\mathrm{C} 0-\mathrm{C} 2$ and FL CMyBP-C (Fig. 3A, C). C0-C2 was much more potent in inhibiting ATPase activity than was FL cMyBP-C $(3.16 \pm 0.18$ vs $6.66 \pm 0.11 \mathrm{mM})$. Although the phosphorylated protein (C0-C2 or FL) was able to shift the inhibition curve to the right, neither cMyBP-C peptide 302A nor 302S enhanced myosin ATPase activity tested at various concentrations (Fig. 3B, D). Based on these observations, we hypothesized that recombinant sarcomere proteins myosin and actin may not be 
adequate to mimic sarcomere function, but rather an intact sarcomere complex structure may be necessary for CMyBP-C peptides to be functional in vitro to enhance ATPase activity.

\section{cMyBP-C peptide 302A and 302S improved ATPase activity in myofibrils}

To test our hypothesis of the role of CMyBP-C on ATPase activity with an intact sarcomere, we isolated myofibrils from the heart samples of sham rats and week 1 post-MI rats where all the sarcomere proteins are present in a native state and are proportional. The integrity of the fully intact sarcomere was confirmed by verifying the presence of the key myofibrillar proteins by gel electrophoreses. All the sarcomere proteins, including the MHC, cMyBP-C, actinin, troponin T and I, as well as the myosin light chain (MLC) proteins, were observed in the gel (Fig. 4A). In addition, we examined the phosphorylation of cMyBP-C p273, p282 and p302 to show a consistent reduction in myofibrils from week 1 post-MI compared to myofibrils isolated from sham animals (Fig. 4B-D). Next, we evaluated the effects of the cMyBP-C peptides in the myofibrillar ATPase assay, which enable the peptides to interact with intact sarcomere proteins (Fig. 5). A significant reduction in ATPase activity of myosin was observed with the myofibrillar proteins isolated from the $\mathrm{Ml}$ infarct region compared to the myofibrillar proteins isolated from sham or Ml-remote regions (sham 159.8 \pm 12.59 vs Ml remote 144.1 $\pm 9.81 \mathrm{vs} \mathrm{Ml} \mathrm{infarct} 67.8 \pm 12.18$, $p<0.005$, Fig. 5A). While none of the peptides (scrambled, 302A or 302S) showed any effect on myofibrillar proteins from the sham hearts, 302A and 302S peptides were able to improve maximal ATPase activity in myofibrils from infarcted hearts at week 1 post-Ml from $60.9 \pm 5.3 \mathrm{nmol} \mathrm{Pi} / \mathrm{min} / \mathrm{mg}$ (sham, $\mathrm{n}=15)$ to $87.9 \pm 7.1 \mathrm{nmol} \mathrm{Pi} / \mathrm{min} / \mathrm{mg}(302 \mathrm{~A}, \mathrm{n}=12 ; p<0.05)$ and $91.3 \pm 6.2 \mathrm{nmol} \mathrm{Pi} / \mathrm{min} / \mathrm{mg}(302 \mathrm{~S}$, $\mathrm{n}=12 ; p<0.05)$, respectively.

Next, we also investigated the ATPase activity and the role of cMyBP-C in myofibrils isolated from a cMyBP-C-transgenic mouse model (AAA and DDD mice) (Fig. 6). Myosin ATPase activity was significantly upregulated in myofibrils from DDD mice $(131.2 \pm 9.33 \mathrm{nmol} \mathrm{Pi} / \mathrm{min} / \mathrm{mg})$ compared to myofibrils from NTG $(93.98 \pm 7.85 \mathrm{nmol} \mathrm{Pi} / \mathrm{min} / \mathrm{mg}, \mathrm{p}<0.01)$ and AAA $(71.06 \pm 6.25 \mathrm{nmol} \mathrm{Pi} / \mathrm{min} / \mathrm{mg}) \mathrm{mice}$ (Fig. 6A). Consistent with the sham data, none of the peptides (scrambled, 302A and 302S) were able to improve ATPase activity in myofibrils from NTG mice (Fig. 6B). On the other hand, both peptides 302A and 302S showed an increased maximal ATPase activity from $62.74 \pm 4.27 \mathrm{nmol} \mathrm{Pi} / \mathrm{min} / \mathrm{mg}$ (control, $\mathrm{n}=11)$ and $58.58 \pm 6.67 \mathrm{nmol} \mathrm{Pi} / \mathrm{min} / \mathrm{mg}$ (scrambled, $\mathrm{n}=10)$ to $94.29 \pm 11.01 \mathrm{nmol} \mathrm{Pi} / \mathrm{min} / \mathrm{mg}(302 \mathrm{~A}, \mathrm{n}=10)$ and $95.05 \pm 10.02 \mathrm{nmol} \mathrm{Pi} / \mathrm{min} / \mathrm{mg}(302 \mathrm{~S}, \mathrm{n}=10)$, representing $61 \%(302 \mathrm{~A})$ and $62 \%(302 \mathrm{~S})$ increase from scrambled, respectively (Fig. 6C). Both cMyBP-C peptides 302A and 302S did not affect calcium sensitivity in either MI or AAA pre-clinical HF models.

\section{CMyBP-C peptide 302A and 302S improved papillary muscle force generation}

To determine whether cMyBP-C synthetic peptides impact sarcomere function, $p$ Ca-force relationship was measured in papillary muscles from AAA and DDD mouse hearts at 3 months of age compared to agematched NTG controls (Fig. 7). Consistent with the myofibril ATPase activity measurements, papillary muscle force measurements indicated an increased maximal force with 302A and 302S in papillary muscles from AAA mice. In addition, the effects of cMyBP-C peptides appear to be dose-dependent (Supp 
Fig. 5). To further characterize the effects of $302 \mathrm{~A}$ and $302 \mathrm{~S}$ peptides on cardiac function, we performed a force redevelopment rate $\left(k_{\mathrm{tr}}\right)$ measurement as an indirect measure of relaxation. The 302A peptide improved $k_{\mathrm{tr}}$ activity while $302 \mathrm{~S}$ did not impact $k_{\mathrm{tr}}$ function, indicating that the latter only directly affects contractility and not relaxation. Albeit to force development effects, the improvement effects on $k_{\mathrm{tr}}$ were also observed with 302A at $25 \mu \mathrm{M}$ (Supp Fig. 5).

\section{Discussion}

CMyBP-C is a key regulator of cardiac contractility, with more than 300 mutations in the MYBPC 3 gene directly linked to the development of cardiomyopathy and heart failure ${ }^{25-27}$. Although the phosphorylation of cMyBP-C has been shown to significantly decrease under pathological conditions in preclinical and clinical models, the dynamic change of cMyBP-C expression and its importance in cardiac kinetics at different time points has not been reported. In this study we carefully characterized the dynamic change of CMyBP-C phosphorylation during the development of HF in a rat MI model. CMyBP-C phosphorylation decreased significantly within week 1 (on day 1 and day 3 post-MI, Fig. 1) after cardiac injury and increased significantly in later time points (week 4 and week 8 post-MI, Supp Fig. 1), which suggests a compensatory effect in the heart. In addition, we observed that the total cMyBP-C also decreases at different time points in the MI pre-clinical HF model. These findings suggest that the cMyBP$\mathrm{C}$ phosphorylation state has a temporal role, and when combined with cMyBP-C expression, contributes to HF development. Therefore, it is important to evaluate any intervention approaches targeting cMyBP-C at relevant time points based on our findings from the preclinical models such as the rat MI model. In addition, the acute loss of total CMyBP-C in the bloodstream is consistent with previous studies, suggesting that $\mathrm{CMyBP}-\mathrm{C}$ could serve as a potential diagnostic biomarker of $\mathrm{Ml}$ and a prognostic marker of $\mathrm{HF}^{28}$. On the other hand, we showed that in human HCM and DCM patients CMyBP-C phosphorylation is linked to the development of disease progression. In HCM patients, both p273 and p282 were reduced, indicating a more general decrease in cMyBP-C phosphorylation (Fig. 2). However, in DCM patients, only p282 was significantly reduced but p273 was maintained at a level similar to healthy donors. Although p302 was not examined due to limited patient samples, our data indicated that the different phosphorylation sites could be impacted in HCM and DCM patients.

At the molecular level, cMyBP-C and myosin interact and regulate the cross-bridge formation required for normal contraction through the phosphorylation ${ }^{29}$. Targeting the phosphorylation site on cMyBP-C with a peptide designed to disrupt its interaction with myosin improves cardiac function. Therefore, we tested a cMyBP-C-derived peptide containing the motif responsible for interaction with myosin that then disrupts the cMyBP-C-myosin association as required for therapeutic action. To fully characterize the effects of peptides on modulating $\mathrm{CMyBP}-\mathrm{C}$, we evaluated the peptides in three different recombinant in vitro and ex-vivo assays: a steady state ATPase, a myofibrils ATPase, and in papillary muscles. In the steady state ATPase assay, no change in ATPase activity was observed in any of the treatment groups (Fig. 3). However, when myofibril proteins were isolated from diseased models (the rat MI model or the mouse cMyBP-C ${ }^{\text {AAA }}$ model), a consistent improvement of ATPase activity was observed in 302S/302A peptide- 
treated groups (Fig. 5, 6). This improvement in ATPase activity was consistent with the improvement of contractility using papillary muscle fibers isolated from cMyBP-C ${ }^{A A A}$ transgenic mice (Fig. 7). Together, this data provided strong evidence that it is critical to evaluate cMyBP-C-targeted therapies under pathological conditions. Additionally, because the data showed the dependence of an intact sarcomere on the activity of peptides, it is clear that full sarcomere function is necessary to properly capture cMyBP$\mathrm{C}$ kinetics. Due to limited permeability of the peptides, immunolocalization of the peptides on intact cardiac myocytes or sarcomeres was not feasible.

Since we observed the improved cardiac effects of peptides in HF models that showed reduced cMyBP-C expression or phosphorylation, we hypothesize that the peptides probably interrupt the interaction of cMyBP-C and myosin, thus improving the myosin head interaction with the thin filament. Under physiological conditions (NTG/DDD or normal), phosphorylated cMyBP-C facilitates the activation of cross-bridge cycling, tethering the thick and thin sarcomeric filaments (Fig. 8; left panel). When cMyBP-C was dephosphorylated in diseased conditions (AAA or HF), it strongly interacts with myosin, thereby preventing its force-generating interaction with actin (Fig. 8; middle panel). The cMyBP-C peptides were able to disrupt the tight interaction between $\mathrm{CMyBP}-\mathrm{C}$ and myosin, hence releasing the thick filaments to resume interacting with the thin filaments to improve muscle contractility (Fig. 8; right panel).

The relevance of $\mathrm{CMyBP}-\mathrm{C}$ phosphorylation has been reported in multiple transgenic rodent models by substituting serine of the phosphorylation sites to mimic activated cMyBP-C, such as the DDD transgenic mouse model ${ }^{17,18}$. Reduction in phosphorylation sites in CMyBP-C can contribute to a decrease in protein expression. We observed the reduced phosphorylation correlated with reduced total cMyBP-C expression (Fig. 1). Recently gene therapy efforts have been reported to deliver MYBPC3 cDNA to improve cardiac function. It has been reported that a single systemic dose of AAV9-carrying MYBPC3 CDNA was able to restore $M Y B P C 3$ mRNA and protein levels and prevent the development of left ventricular hypertrophy (LVH) in MYBPC3-targeted knock-in mice with only $20 \%$ cMyBP-C protein ${ }^{30}$. Another study reported that AAV6-carrying MYBPC3 cDNA was able to achieve similar improvement in engineered 3D heart tissue 31,32. In addition, AAV9-carrying MYBPC3 CDNA has shown to be able to suppress the development of hypertrophy in human iPSC-derived cardiomyocytes from HCM patients ${ }^{33}$. However, the sustained usage of AAV9 as therapy could have drawbacks such as the lack of understanding of cellular and whole-body disposition, dose-exposure relationship, and exposure-response relationship; and a lack of understanding of how immunogenicity affects these important properties ${ }^{34}$. Therefore, the search for specific molecules targeting $\mathrm{CMyBP}-\mathrm{C}$ and its protein-protein interaction is critical to bring effective therapy from bench to bedside. The limitation of this study is that the CMyBP-C peptides have poor permeability and stability, which limited our tests in only the in vitro and ex vivo settings in which we used myofibril proteins and permeabilized papillary muscle fibers. Further studies in an in vivo setting with permeable peptides and good stability would enable a more comprehensive understanding of the mechanism of the cMyBP-C modulator in cardiac function.

\section{Methodology}




\section{Myocardial Infarction Rat Model}

The myocardial infarction (MI)rat model has been previously characterized ${ }^{35}$. Twenty-eight male Sprague-Dawley (SD) rats (Charles River Laboratories, Wilmington, MA) ranging from 8-10 weeks in age and weighing 180-250 $\mathrm{g}$ were used to induce Ml by left anterior descending coronary artery (LAD) ligation. We have followed the ARRIVE guidelines throughout the study for handling animals. All procedures were approved by the Institutional Ethics Committee for Use and Care of Laboratory Animals at Merck \& Co., Inc., Kenilworth, NJ, USA. Briefly, the animals went through left-sided thoracotomy under anesthesia. The pericardial sac was cut open and LAD was identified. A coronary artery occluder was placed loosely around the LAD at $\sim 2 \mathrm{~mm}$ below the origin, and then the occluder was pulled to ligate the coronary artery. Infarction was defined by blanching and discoloration of the left ventricle and ST elevation on the ECG. Then the chest was closed. The animals were closely monitored for surgical complications post-surgery. Twenty-three male SD rats with similar age and body weight were assigned as sham animals who received similar thoracotomy to access the heart and the wound closure but without LAD ligation. Post-MI evaluation to confirm infarcts was performed by echocardiography on day 3 , week 1 , week 4 and week 8 . Meanwhile, the following global cardiac functional parameters were measured: LV end systolic and diastolic inner diameter $(\mathrm{mm}), \mathrm{LV}$ end systolic and diastolic volume $(\mu \mathrm{L})$, stroke volume $(\mu \mathrm{L})$, cardiac output $(\mathrm{mL} / \mathrm{min})$, and heart rate (BPM) (Sup. Fig. 1). The animals with smallsize infarct and ejection fraction $(E F)>40 \%$ were excluded from the study. There were four termination time points: day 3 , week 1 , week 4 , and week 8 . At each termination time point, rats were anesthetized, and the hearts were excised, snap-frozen in liquid nitrogen, and stored at $-80^{\circ} \mathrm{C}$ until use.

\section{CMyBP-C Transgenic Mouse Model}

Previously described cardiac-specific transgenic phospho-ablated (AAA) and phospho-mimetic (DDD) mouse models of $\mathrm{CMyBP}-\mathrm{C}$, with a-myosin heavy chain promoter was used to generate multiple lines of FVB/N mice 6,17,18,20. In brief, the known key phosphorylation sites (Ser-273, -282 and -302) on cMyBP-C and two neighboring potentially alternative phosphorylation sites (Thr-272 and -281) were converted to alanine (A) and aspartic acid (D) residues using standard PCR-based methods. All experiments were conducted under institutional guidelines and approved by the University of Cincinnati Animal Care and Use Committee. Adult mice 12-14 weeks of age were used in this study.

\section{cMyBP-C Peptides}

The cMyBP-C inhibitor peptides were synthesized at the research laboratories of Merck \& Co., Inc., Kenilworth, NJ, USA. All peptides were characterized by high-resolution mass spectrometry-confirmed purity values near or above $90 \%$, and accurate mass values in line with those expected based on the sequences. The sequences of the four original peptides used in this study are listed in Table I. In addition, each peptide was attached with a FITC label to study its localization with sarcomere proteins in cardiomyocytes using an immunostaining technique. Purity and accurate mass analysis were conducted simultaneously by reverse phase LC-UV/MS using a Waters ACQUITY I-class liquid chromatograph 
(Waters, Milford, MA), interfaced with a Xevo G2-XS QTOF mass spectrometer operating in the ESI+

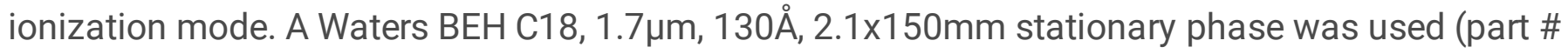
186003556), with $0.1 \%$ trifluoroacetic acid in $\mathrm{H}_{2} \mathrm{O}(\mathrm{A})$ and $0.1 \%$ trifluoroacetic acid in acetonitrile (B) mobile phases. The column was held isothermal at $55^{\circ} \mathrm{C}$, with a linear gradient from $5-55 \%$ B over 25 min, using a $0.4 \mathrm{~mL} / \mathrm{min}$ flow rate.

\section{Western Immunoassay}

The phosphorylation level of the cMyBP-C M-domain at three serine residues (p273, p282, p302) was determined in SD rats subjected to MI surgery using the Western Immunoassay (Wes) procedure (ProteinSimple, Santa Clara, CA). Left ventricle infarct tissue was lysed in a RIPA buffer (Thermo Fisher Scientific, Waltham, MA) supplemented with protease and phosphatase inhibitors. Protein estimation was performed as per kit (Pierce ${ }^{T M}$ BCA Protein Assay Kit 23225, Thermo Fisher Scientific). Samples were prepared as per the Wes protocol, with ProteinSimple recommendations. Antibodies used for the expression study are as follows: Anti-cMyBP-C p273 (1:250), cMyBP-C p-282 (1:250), cMyBP-C p302 (1:250) (all provided through material transfer agreement from Professor Sakthivel Sadayappan, College of Medicine, University of Cincinnati), cMyBP-C total (Santa Cruz Cat \# SC-137182, Lot \# E2913 [1:5 for Wes]), myosin heavy chain (Abcam ab207926 (3-48G5C7), alpha (cardiac) actin (Sigma-Aldrich A9357 clone AC1-20.4.2), Troponin I (Cell Signaling 13083S [D6F8]), and Troponin T (Sigma-Aldrich T6277 Clone JLT-12). . Data was normalized to expressions of housekeeping proteins alpha-actin or hypoxanthine phosphoribosyl transferase (HPRT) to match the protein amount loaded.

\section{Immunohistochemistry}

Immunohistochemistry (IHC) studies were performed using cardiac serial tissue sections from adult human patients (normal subjects, HCM patients, and DCM patients). All methods were carried out in accordance with relevant guidelines and regulations by Nanjing KeyGen BioTech Co., Ltd. and Nanjing GenScript Biotech Co., Ltd. All experimental protocols were in accordance with relevant guidelines and regulations and approved by the Affiliated Drum Tower Hospital, Medical School of Nanjing University. The informed consent was obtained from all subjects and/or their legal guardian by the Affiliated Drum Tower Hospital, Medical School of Nanjing University. Briefly, tissue sections were deparaffinized in xylene and rehydrated through graded alcohol washes prior to incubating with a peroxidase block (3\% $\mathrm{H}_{2} \mathrm{O}_{2}$-methanol) at room temperature for $10 \mathrm{~min}$ to inhibit endogenous peroxidase activity. After washing with phosphate buffered saline (PBS), sections were blocked with $1 \%$ bovine serum albumin (BSA, 50$100 \mu \mathrm{L}$ ) at room temperature for $1 \mathrm{~h}$ to prevent nonspecific binding of the detection reagent. After washing with PBS, sections were incubated with the primary antibody (50-100 $\mu \mathrm{L}$; custom-made rabbit polyclonal antibodies [GenScript, Piscataway, NJ]): p-273 (AFRRTpSLAGAGC), p282 (GAGRRTpSDSHEDAC), and total-protein (AFRRTSLAGAGC) in a humid chamber at $4^{\circ} \mathrm{C}$ overnight. After washing, sections were then incubated with an enhancer (50-100 $\mu \mathrm{L})$ at room temperature for 20 min and then washed with PBS. Sections were then incubated with ImmPRESS HRP PLUS Polymer (Mouse/Rabbit, Vector Laboratories, Burlingame, CA) at room temperature for 20 min, and then washed with PBS. A mixture of 3,3'- 
Diaminobenzidine (DAB) chromogen and substrate was then added at room temperature for about 3-5 min to allow proper color development. The reaction was then terminated by washing the sections with water. Sections were then counterstained with Hematoxylin for 15 min and rinsed with water. Finally, sections were dehydrated in ascending graded alcohol rinse that ended with a xylene wash. Neutral balsam was used to cover the sections. The stained slides were imaged with a microscope at either 200x or 400x magnitude to evaluate protein expression. The positive expression of phospho-proteins was determined by the ratio of the positive region to the total area and was normalized using values of total protein expression.

\section{Recombinant Steady-state ATPase Assay}

A steady-state ATPase assay was performed by measuring inorganic phosphate release using the

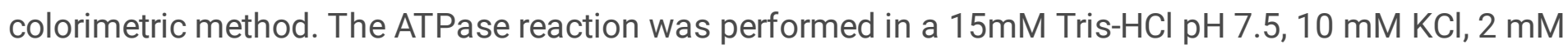
$\mathrm{MgCl}_{2}, 0.1 \mathrm{mM}$ EGTA reaction buffer. Myosin and $\mathrm{FL}$ cMyBP-C and $\mathrm{CO}-\mathrm{C} 2$ domain were made at the research laboratories of Merck \& Co., Inc., Kenilworth, NJ, USA with modifications according to references

36. Actin was made from rabbit skeletal muscle at the research laboratories of Merck \& Co., Inc., Kenilworth, NJ, USA with minor modifications ${ }^{37,38}$. All the proteins used in the assay were diluted in a reaction buffer. A $30 \mu \mathrm{L}$ reaction was set up by adding FL cMyBP-C or truncated cMyBP-C (C0-C2), myosin $(1 \mu \mathrm{M})$, F-actin $(10 \mu \mathrm{M})$ and ATP $(2 \mathrm{mM})$. F-actin was prepared from G-actin by adding $50 \mathrm{mM} \mathrm{KCl}$ and $2 \mathrm{mM} \mathrm{MgCl} 2$ followed by brief vortexing and $30 \mathrm{~min}$ incubation on ice for polymerization. The plate was spun at $2000 \mathrm{rpm}$ for 15 seconds to help reduce bubbles, and the reaction plate was incubated at $37^{\circ} \mathrm{C}$ with constant shaking for $60 \mathrm{~min}$. The reaction was stopped by diluting the proteins $(1: 20)$ in water. $30 \mu \mathrm{L}$ of the 1:20 reaction mix was transferred to a fresh plate and water was added to make up to 200 $\mu \mathrm{L}$ as per kit instructions (Abcam ab65622, Waltham, MA). Phosphate standards were also prepared as per kit instructions. Finally, $30 \mu \mathrm{L}$ of detection reagent was added to all standard and sample wells, incubated at RT for 30 min with constant shaking, and then read at $650 \mathrm{nM}$ endpoint in SpectraMax (Molecular Devices, San Jose, CA).

\section{Myofibrillar ATPase Assay}

Myofibrillar ATPase activity was determined by measuring inorganic phosphate release ${ }^{39}$. Myofibrillar proteins were isolated and purified, as previously reported ${ }^{40}$, and suspended in a F60 buffer with $500 \mathrm{mM}$ $\mathrm{NaCl}$. These proteins were extracted from week 1 post-MI and sham rat heart samples, as well as cMyBP$C$ transgenic mice (NTG, AAA, and DDD) heart samples. Protein was then determined by Coomassie blue staining (Bio-Rad Laboratories, Hercules, CA) of the SDS-PAGE gels (Fig. 4). The reaction mixture contained 0.25-0.5 mg/mL myofibrillar proteins, $15 \mathrm{mM}$ Tris- $\mathrm{HCl}, 10 \mathrm{mM} \mathrm{KCl}, 2 \mathrm{mM} \mathrm{MgCl} 2,0.5 \mathrm{mM}$ EGTA, and $5 \mathrm{mM}$ ATP ( $\mathrm{pH}$ 7.0). Assays were performed in 96-well microtiter plates at $p \mathrm{Ca}^{2+}$ concentrations $(p \mathrm{Ca})$ from $8.0-2.0$ at $37^{\circ} \mathrm{C}$. The $120-\mu \mathrm{L}$ reaction mixture was incubated at $37^{\circ} \mathrm{C}$ for $15 \mathrm{~min}$ and centrifuged at $1500 \mathrm{rpm}$ for $3 \mathrm{~min}$. Each $30 \mu \mathrm{L}$ of supernatant was transferred into a new 96-well microtiter plate and mixed with $170 \mu \mathrm{L} \mathrm{dH} 2 \mathrm{O}$. A $30 \mu \mathrm{L}$ detection reagent (Phosphate Assay Kit, Abcam) 
was added to create a reaction to develop color after which the production of inorganic phosphate was determined calorimetrically at $625 \mathrm{nM}$ using a kinetic microplate reader (Molecular Devices). $\mathrm{Ca}^{2}+-$ stimulated ATPase activity was calculated by subtracting the activity at $p \mathrm{Ca} 2.0$ from the activity at $p \mathrm{Ca}$ 8.0.

\section{Papillary Muscle Assay}

Whole hearts were rapidly excised and washed with 1X Krebs-Henseleit Buffer (Sigma-Aldrich, St. Louis, $\mathrm{MO}$ ), followed by the addition of $1 \mathrm{~g}$ D-glucose, $0.84 \mathrm{~g} \mathrm{NaHCO}$, and $0.76 \mathrm{~g} \mathrm{BDM}$. Each heart was cut to carefully expose the papillary muscles in the LV. As previously described ${ }^{20}$, the papillary muscles were excised under a dissecting scope (V8 Stereo, PlanAPO S 0.63x FWD $81 \mathrm{~mm}$, Zeiss, Dublin, CA) and permeabilized overnight in $1 \%$ Triton $X-100$ prediluted to $10 \%$ in mounting relaxing solution $(97.92 \mathrm{mM}$ $\mathrm{KOH}, 6.24 \mathrm{mM}$ ATP, $10 \mathrm{mM}$ EGTA, $10 \mathrm{mM} \mathrm{Na2CrP,} 47.58 \mathrm{mM}$ potassium propionate, $100 \mathrm{mM}$ BES, 6.54 $\mathrm{mM} \mathrm{MgCl} 2$, and $1 \mathrm{mM} \mathrm{DTT}$ ), at $4^{\circ} \mathrm{C}$ to remove the cell membrane and membrane-bound proteins. The papillary muscles were then further trimmed into fiber bundles of approximately $1 \mathrm{~mm}$ in length under the dissecting microscope. Straight fiber bundles were selected based upon uniformity and were then attached at each end with aluminum t-clips. Each fiber bundle was gently washed with a fresh relaxing solution on ice and was used within $12 \mathrm{~h}$. The t-clipped fibers were then attached to a force transducer and a high-speed length controller (Aurora Scientific, Inc., Aurora, ON, Canada). Muscle dimensions (cross-sectional area, length) were determined using an ocular micrometer mounted on the dissection microscope (resolution, $\sim 10 \mu \mathrm{M}$ ). These muscle dimensions were used to normalize contractile force and sarcomere length. The latter was set at $2.0 \mu \mathrm{M}$ and was continuously monitored through Aurora's HighSpeed Video Sarcomere Length (HVSL) measurement system. The strength and rundown of the muscle fiber attachment was determined by exposing the attached fibers to the maximum calcium-saturating activating solution at the beginning and end of the experimental protocol. Developing isometric force was recorded at varying calcium concentrations ( $p$ Ca from10.0 to 4.5 ) in the presence or absence of peptides, and zero baseline force level was subtracted from all force recordings at each activating cycle. All force measurements were corrected for rundown and normalized to the cross-sectional area. Fibers with more than $20 \%$ rundown were excluded from the data analysis. Data were acquired using Aurora's 600A RealTime Muscle Data Acquisition and Analysis System. Each individual force-calcium relationship was fitted to a modified Hill equation (Force/Forcemax $=\left[\mathrm{Ca}^{2+}\right] \mathrm{n} /\left(\mathrm{pCa} 50 \mathrm{n}+\left[\mathrm{Ca}^{2+}\right] \mathrm{n}\right)$ in which $\mathrm{n}$ is the Hill slope. Similarly, the rate of tension redevelopment $\left(k_{\mathrm{tr}}\right)$ was also measured in fibers harvested from AAA, DDD, and NTG heart tissue at $p \mathrm{Ca} 4.5$.

\section{Statistical analysis}

All data are represented as the mean \pm standard error of the mean (SEM), unless otherwise indicated. Statistical analysis was performed using GraphPad Prism (v 6.0, GraphPad Software, San Diego, CA). Data were analyzed using a two-way ANOVA with Tukey's post-hoc test. A $p<0.05$ value was considered to be statistically significant. 


\section{Declarations}

\section{Author Information}

\section{Affiliations}

Cardiometabolic Department, Merck \& Co., Inc., South San Francisco, CA 94080 USA

Luqia Hou, Priti Anand, Yinhong Chen, Nesrine El-Bizri, and Gayathri Swaminath

Analytical R\&D, Merck \& Co., Inc., South San Francisco, CA 94080 USA

Chad J. Pickens

Discovery Chemistry, Merck \& Co., Inc., South San Francisco, CA, 94080, USA

Michael Seganish

Heart, Lung and Vascular Institute, Department of Internal Medicine, Division of Cardiovascular Health and Disease, University of Cincinnati, Cincinnati, OH 45267 USA

Mohit Kumar, Sakthivel Sadayappan

\section{Contributions}

L.H. and M.K. contributed equally to this work.

L.H., M.K., S.S. and G.S. conceived and designed the experiments. L.H., M.K., P.A., Y.C., N.E., and C.P. performed the experiments and analyzed experimental data. M.S. helped to synthesize the peptides. L.H. and M.K. drafted the manuscript. G.S. and S.S. assisted in writing, editing and reviewing the manuscript. All authors read the manuscript.

\section{Corresponding author}

Gayathri Swaminath, PhD., MBA, Cardiometabolic Department

Merck \& Co., Inc., 213 East Grand Ave, South San Francisco, CA 94080 USA;

Phone: +1-(415)-2157009

Email: gswaminath@gmail.com

\section{Funding}

Mohit Kumar was supported by an American Heart Association Predoctoral Fellowship (17PRE33630192). Sakthivel Sadayappan has received support from National Institutes of Health grants R01 AR078001, R01 HL130356, R38 HL155775 and R01 HL143490; American Heart Association 2019 
Institutional Undergraduate Student (19UFEL34380251) and Transformation (19TPA34830084) awards; and has received support from Novo Nordisk, Amgen, AstraZeneca, MyoKardia, and Merck.

\section{Competing Interests}

Sakthivel Sadayappan provided consulting and collaborative research studies to the Leducq Foundation, Pfizer, Novo Nordisk, Amgen, AstraZeneca, MyoKardia but such work is unrelated to the content of this manuscript. The authors declare that they have no conflicts of interest with the contents of this article.

\section{References}

1. Winegrad, S. Cardiac myosin binding protein C. Circ Res 84, 1117-1126, doi:10.1161/01.res.84.10.1117 (1999).

2. Winegrad, S. Myosin binding protein C, a potential regulator of cardiac contractility. Circ Res 86, 6-7, doi:10.1161/01.res.86.1.6 (2000).

3. Barefield, D. \& Sadayappan, S. Phosphorylation and function of cardiac myosin binding protein-C in health and disease. J Mol Cell Cardiol 48, 866-875, doi:10.1016/j.yjmcc.2009.11.014 (2010).

4. Harris, S. P. et al. Hypertrophic cardiomyopathy in cardiac myosin binding protein-C knockout mice. Circ Res 90, 594-601, doi:10.1161/01.res.0000012222.70819.64 (2002).

5. Harris, S. P., Lyons, R. G. \& Bezold, K. L. In the thick of it: HCM-causing mutations in myosin binding proteins of the thick filament. Circ Res 108, 751-764, doi:10.1161/CIRCRESAHA.110.231670 (2011).

6. McNamara, J. W., Singh, R. R. \& Sadayappan, S. Cardiac myosin binding protein-C phosphorylation regulates the super-relaxed state of myosin. Proc Natl Acad Sci U S A 116, 11731-11736, doi:10.1073/pnas.1821660116 (2019).

7. Garvey, J. L., Kranias, E. G. \& Solaro, R. J. Phosphorylation of C-protein, troponin I and phospholamban in isolated rabbit hearts. Biochem J 249, 709-714, doi:10.1042/bj2490709 (1988).

8. Gruen, M. \& Gautel, M. Mutations in beta-myosin S2 that cause familial hypertrophic cardiomyopathy (FHC) abolish the interaction with the regulatory domain of myosin-binding protein-C. J Mol Bio/ 286, 933-949, doi:10.1006/jmbi.1998.2522 (1999).

9. Weisberg, A. \& Winegrad, S. Alteration of myosin cross bridges by phosphorylation of myosin-binding protein C in cardiac muscle. Proc Natl Acad Sci U S A 93, 8999-9003, doi:10.1073/pnas.93.17.8999 (1996).

10. Levine, R., Weisberg, A., Kulikovskaya, I., McClellan, G. \& Winegrad, S. Multiple structures of thick filaments in resting cardiac muscle and their influence on cross-bridge interactions. Biophys $J \mathbf{8 1}$, 1070-1082, doi:10.1016/S0006-3495(01)75764-5 (2001).

11. Hofmann, P. A., Greaser, M. L. \& Moss, R. L. C-protein limits shortening velocity of rabbit skeletal muscle fibres at low levels of Ca2+ activation. J Physiol 439, 701-715, doi:10.1113/jphysiol.1991.sp018689 (1991). 
12. Harris, S. P., Rostkova, E., Gautel, M. \& Moss, R. L. Binding of myosin binding protein-C to myosin subfragment S2 affects contractility independent of a tether mechanism. Circ Res 95, 930-936, doi:10.1161/01.RES.0000147312.02673.56 (2004).

13. Stelzer, J. E., Patel, J. R. \& Moss, R. L. Protein kinase A-mediated acceleration of the stretch activation response in murine skinned myocardium is eliminated by ablation of cMyBP-C. Circ Res 99, 884-890, doi:10.1161/01.RES.0000245191.34690.66 (2006).

14. Stelzer, J. E., Patel, J. R., Walker, J. W. \& Moss, R. L. Differential roles of cardiac myosin-binding protein $\mathrm{C}$ and cardiac troponin I in the myofibrillar force responses to protein kinase $\mathrm{A}$ phosphorylation. Circ Res 101, 503-511, doi:10.1161/CIRCRESAHA.107.153650 (2007).

15. Witayavanitkul, N. et al. Myocardial infarction-induced N-terminal fragment of cardiac myosinbinding protein C (cMyBP-C) impairs myofilament function in human myocardium. J Biol Chem 289, 8818-8827, doi:10.1074/jbc.M113.541128 (2014).

16. Rosas, P. C. et al. Phosphorylation of cardiac Myosin-binding protein-C is a critical mediator of diastolic function. Circ Heart Fail 8, 582-594, doi:10.1161/CIRCHEARTFAILURE.114.001550 (2015).

17. Sadayappan, S. et al. Cardiac myosin-binding protein-C phosphorylation and cardiac function. Circ Res 97, 1156-1163, doi:10.1161/01.RES.0000190605.79013.4d (2005).

18. Sadayappan, S. et al. Cardiac myosin binding protein $\mathrm{C}$ phosphorylation is cardioprotective. Proc Natl Acad Sci U S A 103, 16918-16923, doi:10.1073/pnas.0607069103 (2006).

19. Sadayappan, S. et al. A Critical Function for Ser-282 in Cardiac Myosin Binding Protein-C Phosphorylation and Cardiac Function. Circulation Research 109, 141-U146, doi:10.1161/Circresaha.111.242560 (2011).

20. Kumar, M. et al. Cardiac Myosin-binding Protein C and Troponin-I Phosphorylation Independently Modulate Myofilament Length-dependent Activation. J Biol Chem 290, 29241-29249, doi:10.1074/jbc.M115.686790 (2015).

21. Tong, C. W., Stelzer, J. E., Greaser, M. L., Powers, P. A. \& Moss, R. L. Acceleration of crossbridge kinetics by protein kinase A phosphorylation of cardiac myosin binding protein $\mathrm{C}$ modulates cardiac function. Circ Res 103, 974-982, doi:10.1161/CIRCRESAHA.108.177683 (2008).

22. Rosas, P. C. et al. Cardiac Myosin Binding Protein-C Phosphorylation Mitigates Age-Related Cardiac Dysfunction: Hope for Better Aging? JACC Basic Transl Sci 4, 817-830, doi:10.1016/j.jacbts.2019.06.003 (2019).

23. McConnell, B. K. et al. Dilated cardiomyopathy in homozygous myosin-binding protein-C mutant mice. J Clin Invest 104, 1771, doi:10.1172/jci7377c1 (1999).

24. Richard L. Moss, A. F. Inhibition of MYBP-C Binding to Myosin as a Treatment for Heart Failure. United States patent (2015).

25. Ingles, J. \& Semsarian, C. Making the case for cascade screening among families with inherited heart disease. Heart Rhythm 17, 113-114, doi:10.1016/j.hrthm.2019.07.014 (2020).

26. Carrier, L., Mearini, G., Stathopoulou, K. \& Cuello, F. Cardiac myosin-binding protein C (MYBPC3) in cardiac pathophysiology. Gene 573, 188-197, doi:10.1016/j.gene.2015.09.008 (2015). 
27. Behrens-Gawlik, V., Mearini, G., Gedicke-Hornung, C., Richard, P. \& Carrier, L. MYBPC3 in hypertrophic cardiomyopathy: from mutation identification to RNA-based correction. Pflugers Arch 466, 215-223, doi:10.1007/s00424-013-1409-7 (2014).

28. Govindan, S. et al. Cardiac myosin binding protein-C is a potential diagnostic biomarker for myocardial infarction. J Mol Cell Cardio/ 52, 154-164, doi:10.1016/j.yjmcc.2011.09.011 (2012).

29. Sadayappan, S. \& de Tombe, P. P. Cardiac myosin binding protein-C: redefining its structure and function. Biophys Rev 4, 93-106, doi:10.1007/s12551-012-0067-x (2012).

30. Mearini, G. et al. Mybpc3 gene therapy for neonatal cardiomyopathy enables long-term disease prevention in mice. Nat Commun 5, 5515, doi:10.1038/ncomms6515 (2014).

31. Wijnker, P. J. et al. Comparison of the effects of a truncating and a missense MYBPC3 mutation on contractile parameters of engineered heart tissue. J Mol Cell Cardiol 97, 82-92, doi:10.1016/j.yjmcc.2016.03.003 (2016).

32. Dutsch, A. et al. Phosphomimetic cardiac myosin-binding protein $\mathrm{C}$ partially rescues a cardiomyopathy phenotype in murine engineered heart tissue. Sci Rep 9, 18152, doi:10.1038/s41598019-54665-2 (2019).

33. Prondzynski, M. et al. Evaluation of MYBPC3 trans-Splicing and Gene Replacement as Therapeutic Options in Human iPSC-Derived Cardiomyocytes. Mol Ther Nucleic Acids 7, 475-486, doi:10.1016/j.omtn.2017.05.008 (2017).

34. Chowdhury, E. A. et al. Current progress and limitations of AAV mediated delivery of protein therapeutic genes and the importance of developing quantitative pharmacokinetic/pharmacodynamic (PK/PD) models. Adv Drug Deliv Rev 170, 214-237, doi:10.1016/j.addr.2021.01.017 (2021).

35. Wu, Y., Yin, X., Wijaya, C., Huang, M. H. \& McConnell, B. K. Acute myocardial infarction in rats. J Vis Exp, doi:10.3791/2464 (2011).

36. Nag, S. et al. The myosin mesa and the basis of hypercontractility caused by hypertrophic cardiomyopathy mutations. Nat Struct Mol Biol 24, 525-533, doi:10.1038/nsmb.3408 (2017).

37. Rohde, J. A., Thomas, D. D. \& Muretta, J. M. Heart failure drug changes the mechanoenzymology of the cardiac myosin powerstroke. Proc Natl Acad Sci U SA 114, E1796-E1804, doi:10.1073/pnas.1611698114 (2017).

38. Margossian, S. S. \& Lowey, S. Preparation of myosin and its subfragments from rabbit skeletal muscle. Methods Enzymo/ 85 Pt B, 55-71, doi:10.1016/0076-6879(82)85009-x (1982).

39. Krenz, M. et al. Analysis of myosin heavy chain functionality in the heart. J Biol Chem 278, 1746617474, doi:10.1074/jbc.M210804200 (2003).

40. Fewell, J. G. et al. Functional significance of cardiac myosin essential light chain isoform switching in transgenic mice. J Clin Invest 101, 2630-2639, doi:10.1172/JCI2825 (1998).

\section{Tables}


Table I. cMyBP-C peptides sequence and characterization.

\begin{tabular}{llllll} 
Name & Peptide Sequence & $\begin{array}{l}\text { UV } \\
\text { Purity } \\
(\%)\end{array}$ & $\begin{array}{l}\text { Observed } \\
{[\mathbf{M}+]^{+}(\mathbf{D a})}\end{array}$ & $\begin{array}{l}\text { Theoretical } \\
{\left[\mathbf{M}+\mathbf{H}^{+}(\mathrm{Da})\right.}\end{array}$ & $\begin{array}{l}\text { Mass } \\
\text { Accuracy }\end{array}$ \\
\hline Scrambledd & DALFKKAKSLRFELRSRD & 96.4 & 2180.2241 & 2180.2298 & 2.61 \\
\hline 302A & FSSLLKKRDAFRRDAKLE & 93.5 & 2180.2202 & 2180.2298 & 4.4 \\
\hline 302S & FSSLLKKRDSFRRDSKLE & 90.7 & 2212.2175 & 2212.2197 & 0.99 \\
\hline
\end{tabular}

CMyBP-C peptides were generated and characterized by high resolution mass spectrometry. All peptide samples have purity values near or above $90 \%$, with accurate mass values in line with expectation based on the sequences.

\section{Figures}

A
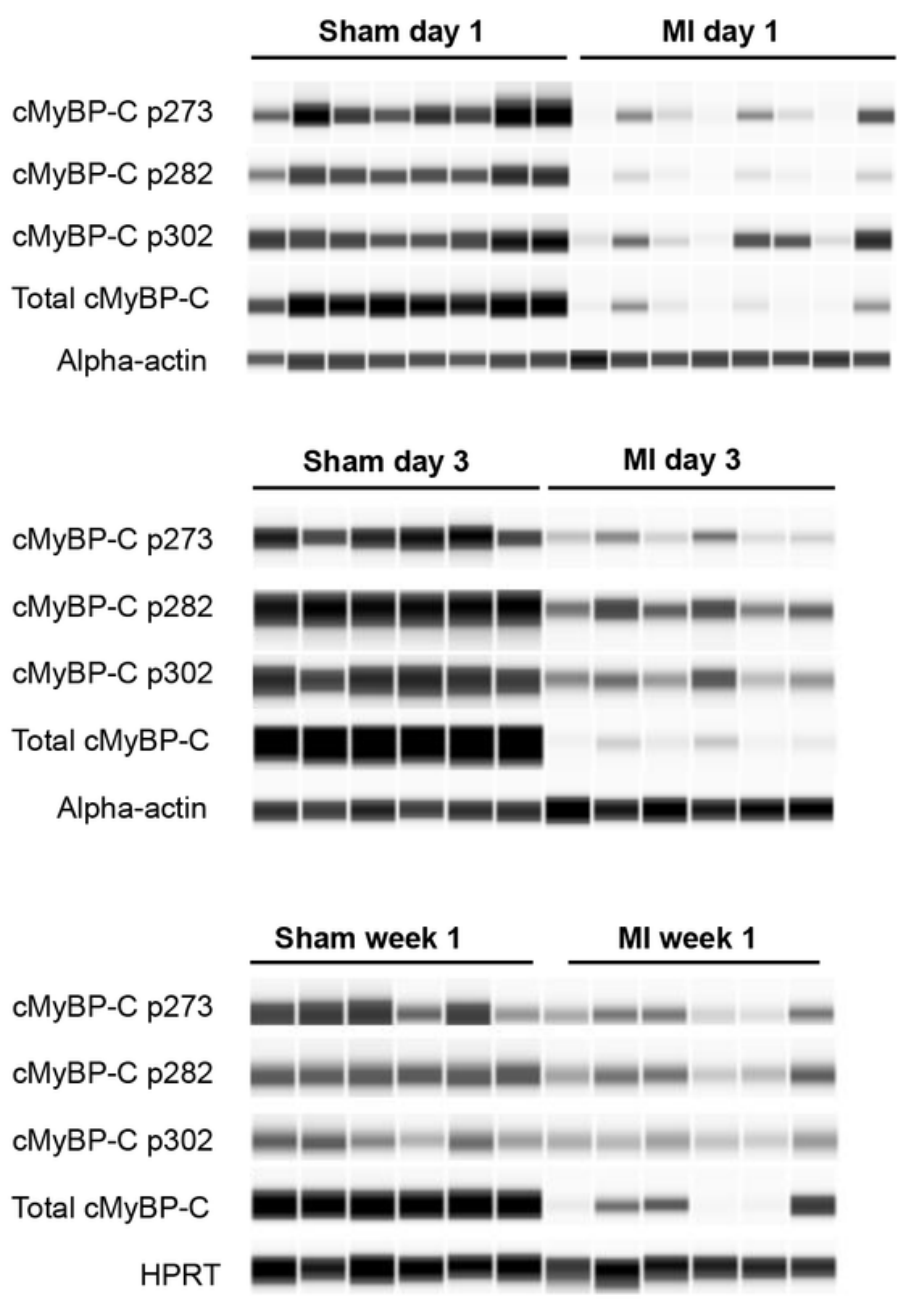

B
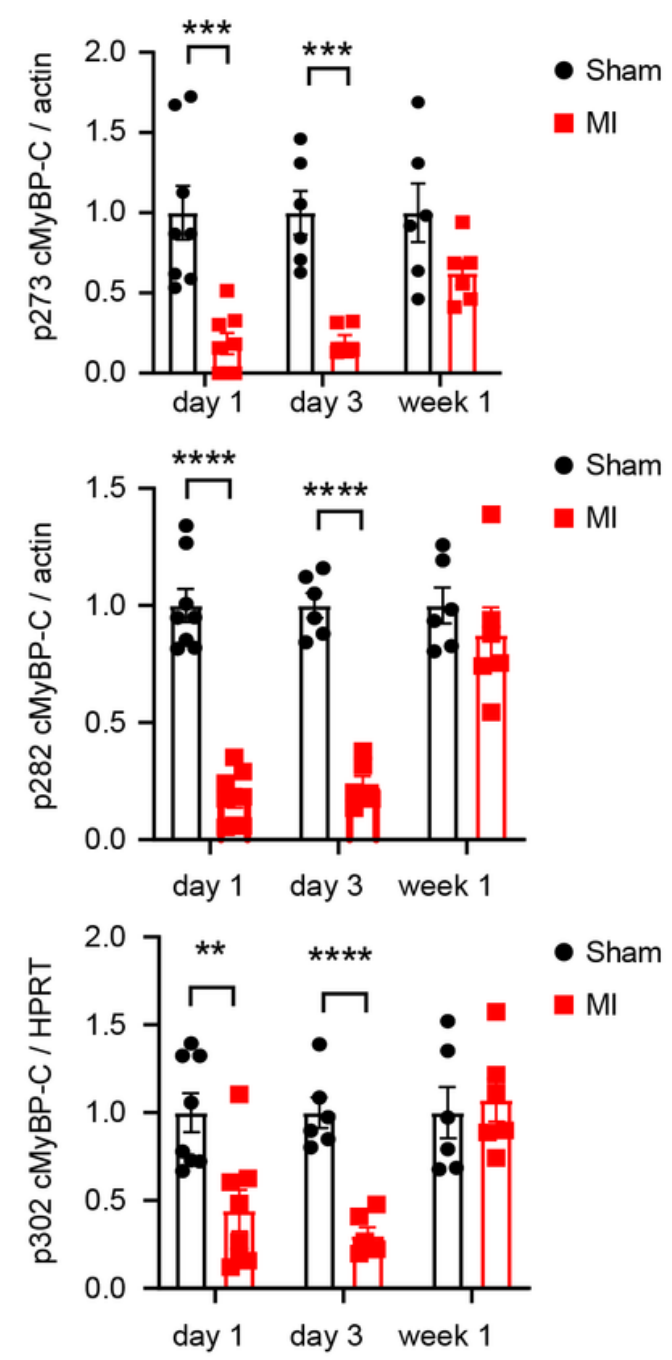

Figure 1 
cMyBP-C phosphorylation was reduced in day 1 and day 3 but not in week 1 post-MI rats compared to sham. (A) Western blot of phosphorylated cMyBP-C tested with site-specific phospho cMyBP-C antibodies p273, p282, p302, total cMyBP-C antibodies, and housekeeping protein antibodies alpha-actin or HPRT.

(B) Quantification of cMyBP-C p273, p282, and p302 normalized to housekeeping proteins. Data represent means \pm SEM $(N=6-8) .{ }^{* \star} p<0.01 ;{ }^{* \star \star} p<0.005 ; * \star \star \star x<0.001$; unpaired two-tailed Student's t-test.
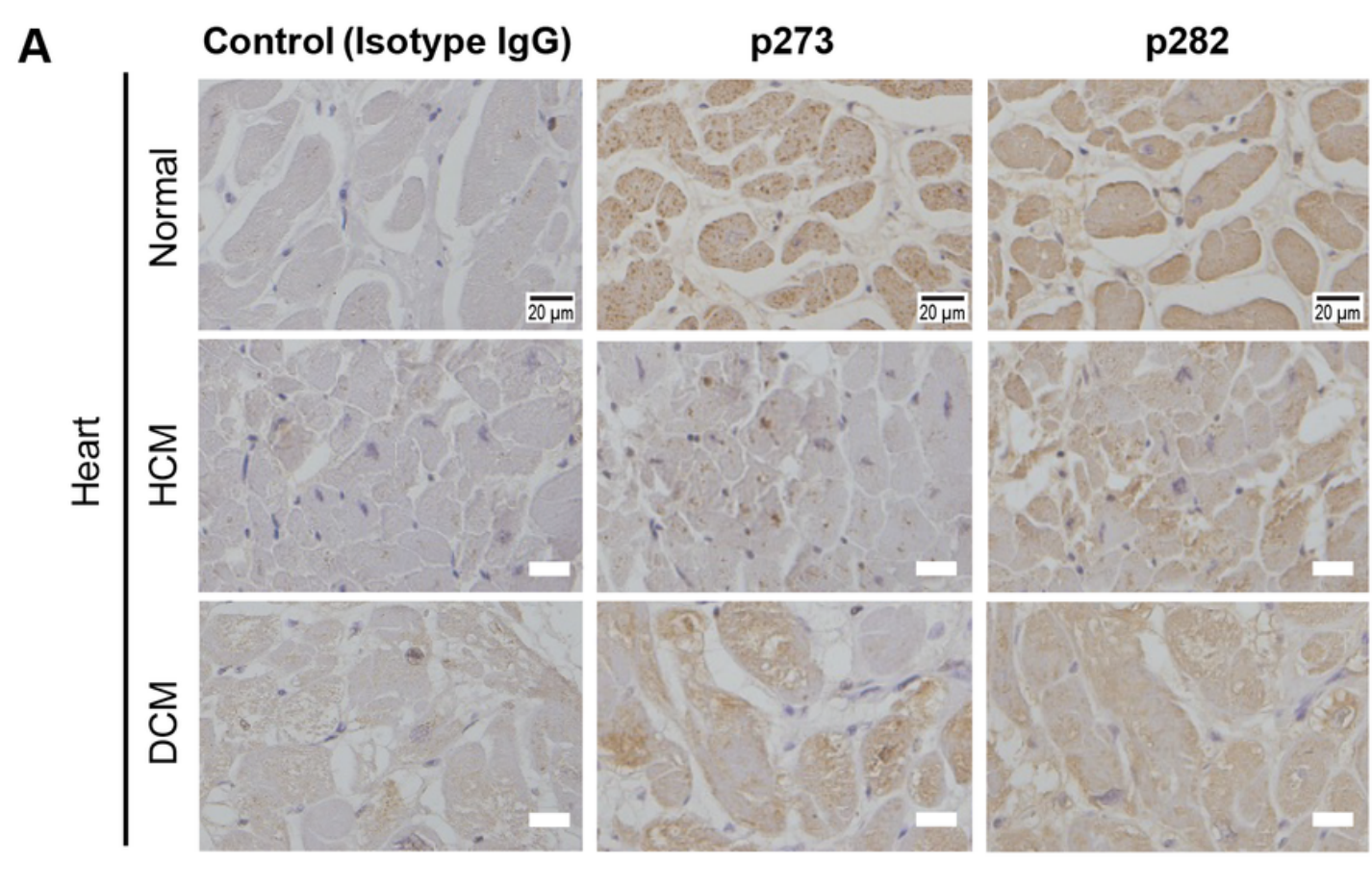

Total

B

p273/Total

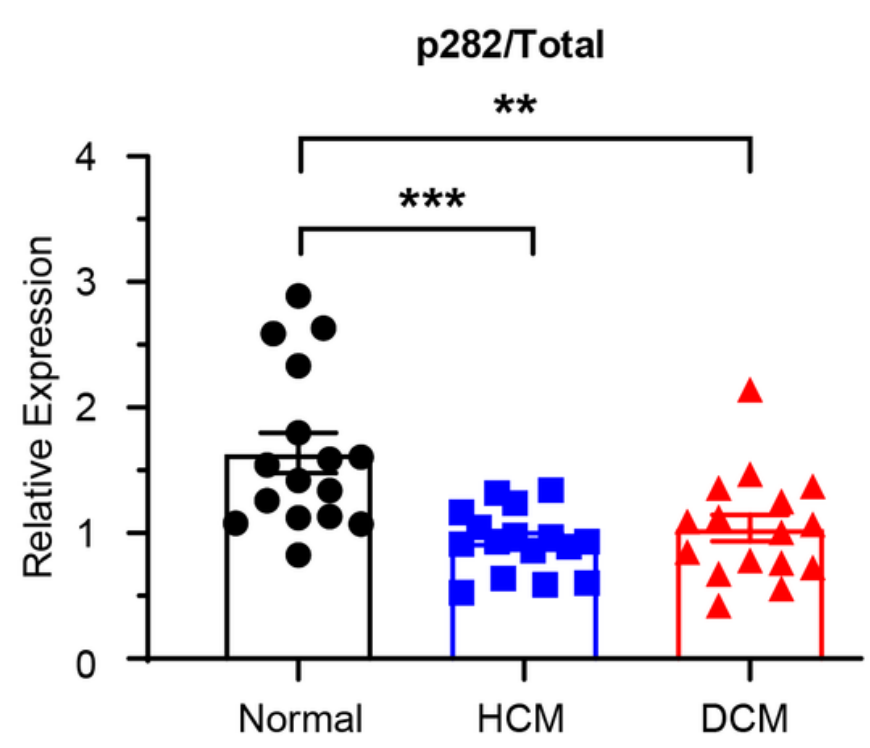

Figure 2

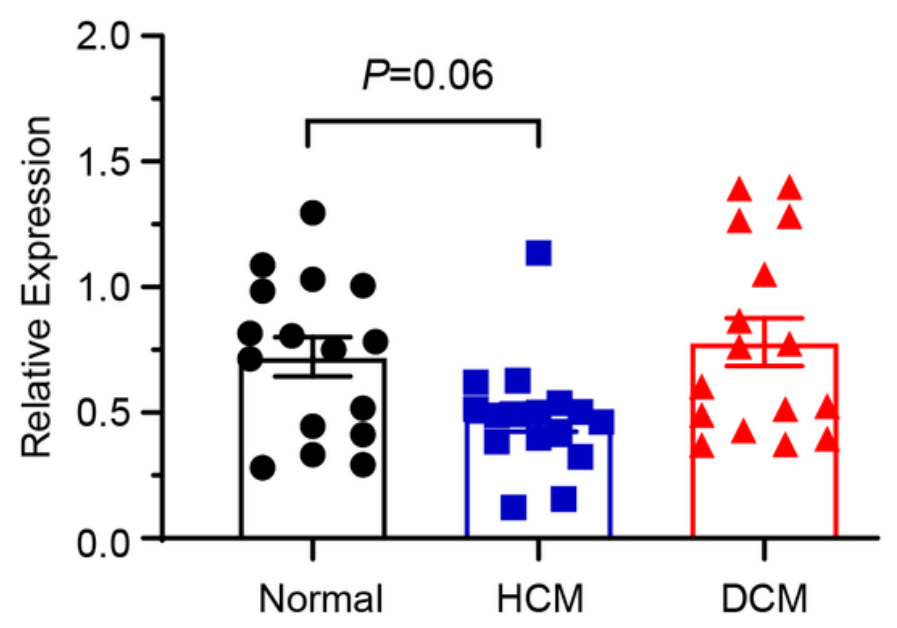

Immunohistochemistry of cardiac tissue sections from HCM and DCM patients showed reduced cMyBP-C phosphorylation compared to healthy donors. (A) IHC images of p273 and p282 in normal, HCM, and DCM heart samples. (B) Corresponding quantifications of p273 and p282 in all heart samples. P273 and p282 values were normalized to total cMyBP-C values. Data represent mean $\pm S E M(N=4$ patients, $n=16$ 
images). The scale bar in figures represents $20 \mu \mathrm{M}$. DCM, dilated cardiomyopathy; HCM, hypertrophic cardiomyopathy: IHC, immunohistochemistry. ${ }^{\star *} \mathrm{P}<0.01$; ${ }^{* \star \star} \mathrm{P}<0.005$; one-way ANOVA with Tukey posttest.
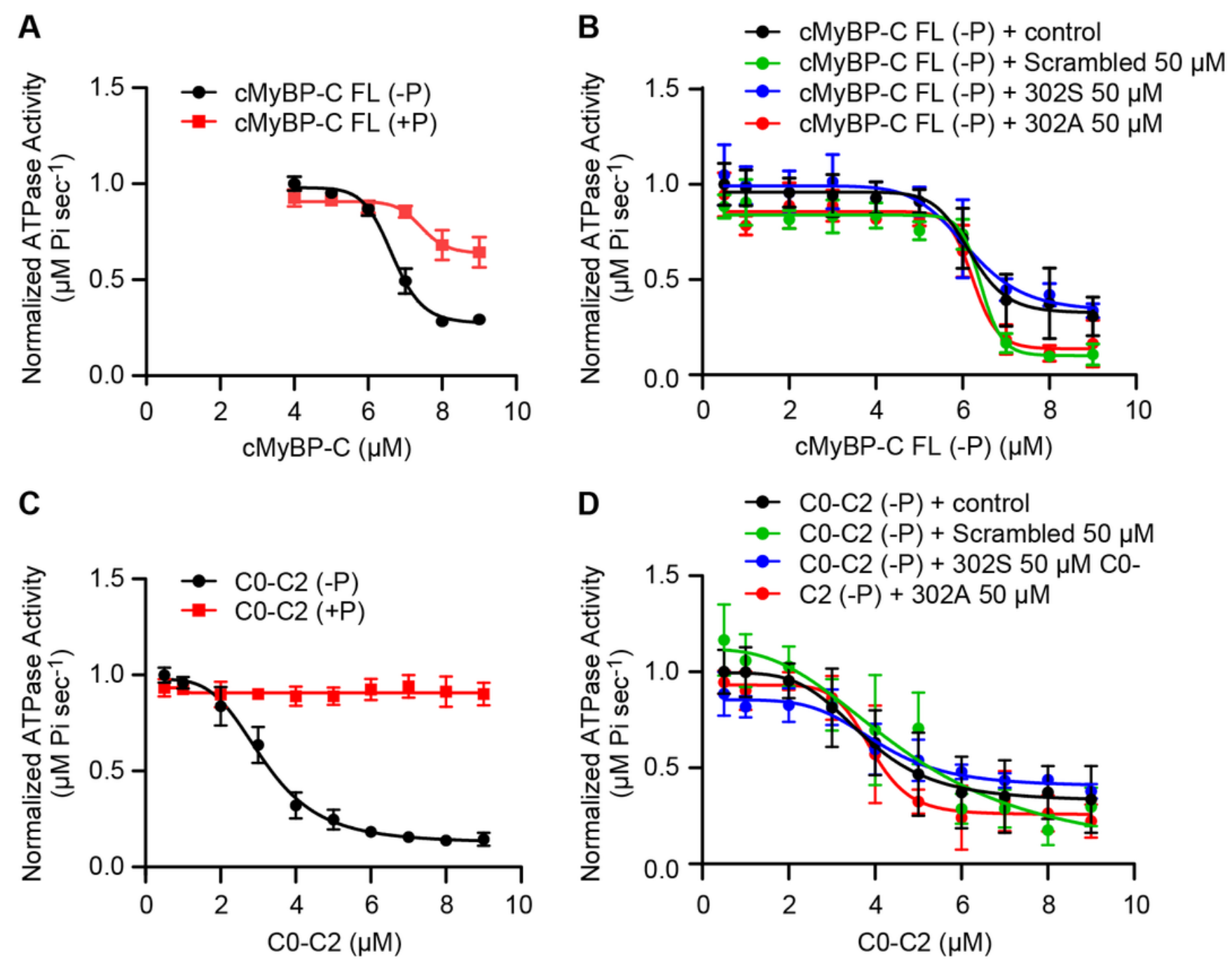

Figure 3

CMyBP-C peptides have no effect in steady-state ATPase assay with truncated CMyBP-C COC2 or FL cMyBP-C. (A) Unphosphorylated FL cMyBP-C inhibited myosin ATPase activity more than did phosphorylated cMyBP-C. (B) cMyBP-C peptide 302A or 302S had no effect on myosin ATPase FL CMYBP-C activity. (C) Unphosphorylated cMyBP-C COC2 inhibited myosin ATPase activity but not the phosphorylated cMyBP-C. (D) CMyBP-C peptide 302A or 302S had no effect on CO-C2 myosin ATPase activity. Data represent mean \pm SEM $(\mathrm{N}=4-12)$. $\mathrm{FL}$, full-length. 

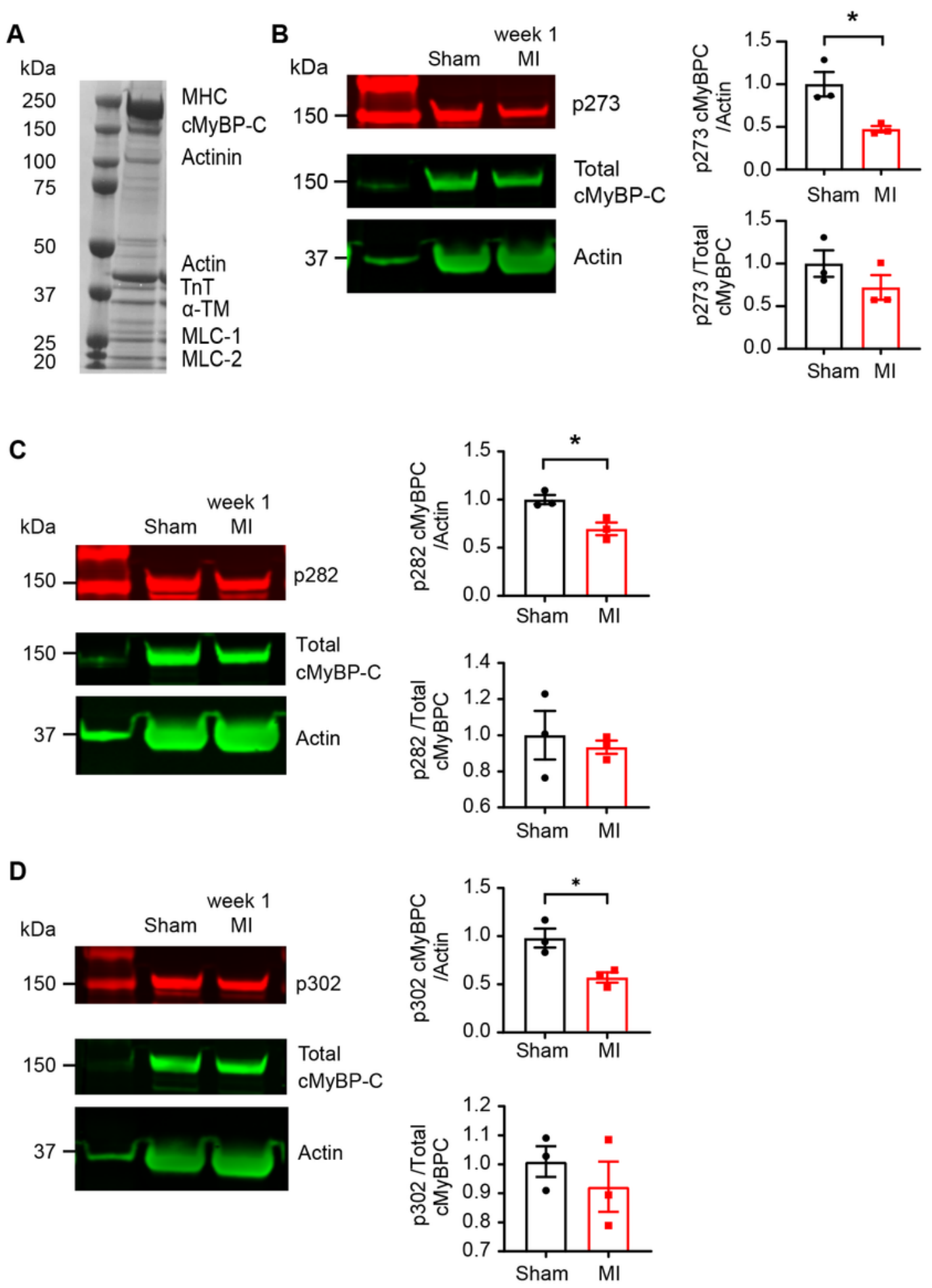

\section{Figure 4}

CMyBP-C phosphorylation was reduced in myofibrils from week 1 post-MI rats compared to sham. (A) Coomassie Blue staining of sarcomere proteins in isolated myofibrils from sham rats. (B-D) Representative Western blot analysis using site-specific phospho-cMyBP-C antibodies p273, p282, and p302 in sham and MI myofibrils. Quantification was performed using either housekeeping protein (actin), 
or total cMyBP-C. Data represent mean \pm SEM $(\mathrm{N}=3) .{ }^{*} \mathrm{p}<0.05$; unpaired two-tailed Student's t-test. MI, myocardial infarction.

A
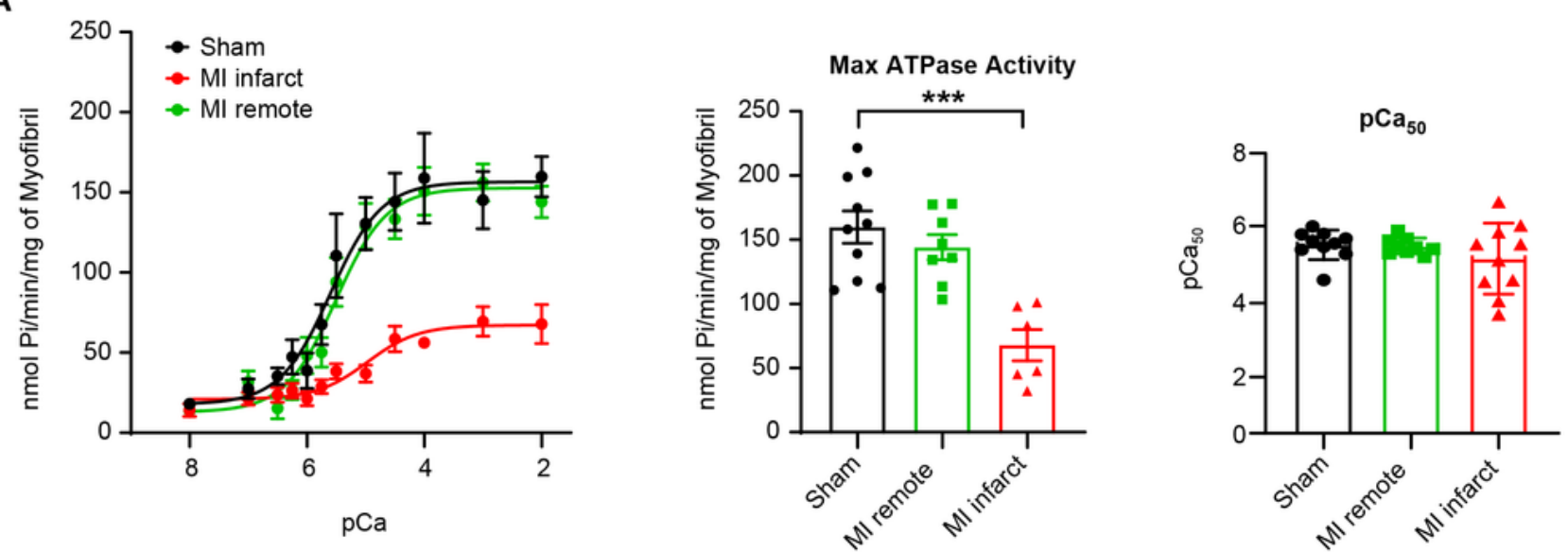

B
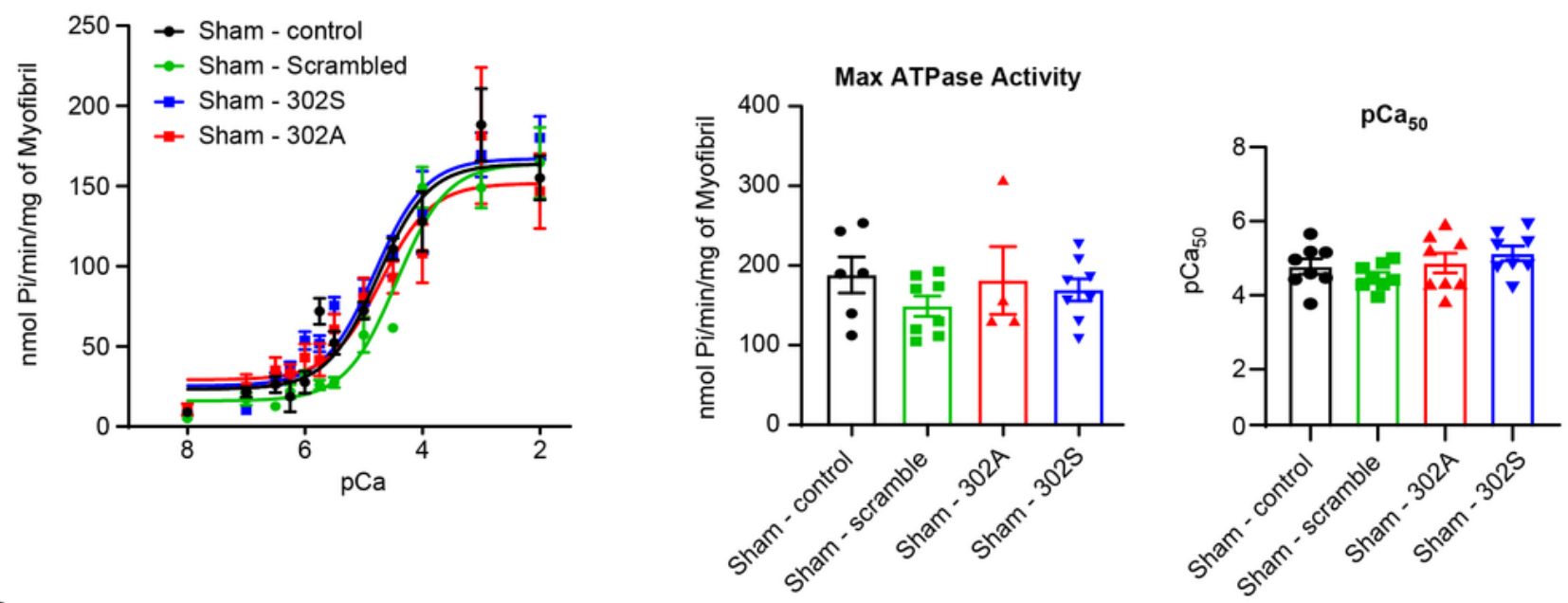

C
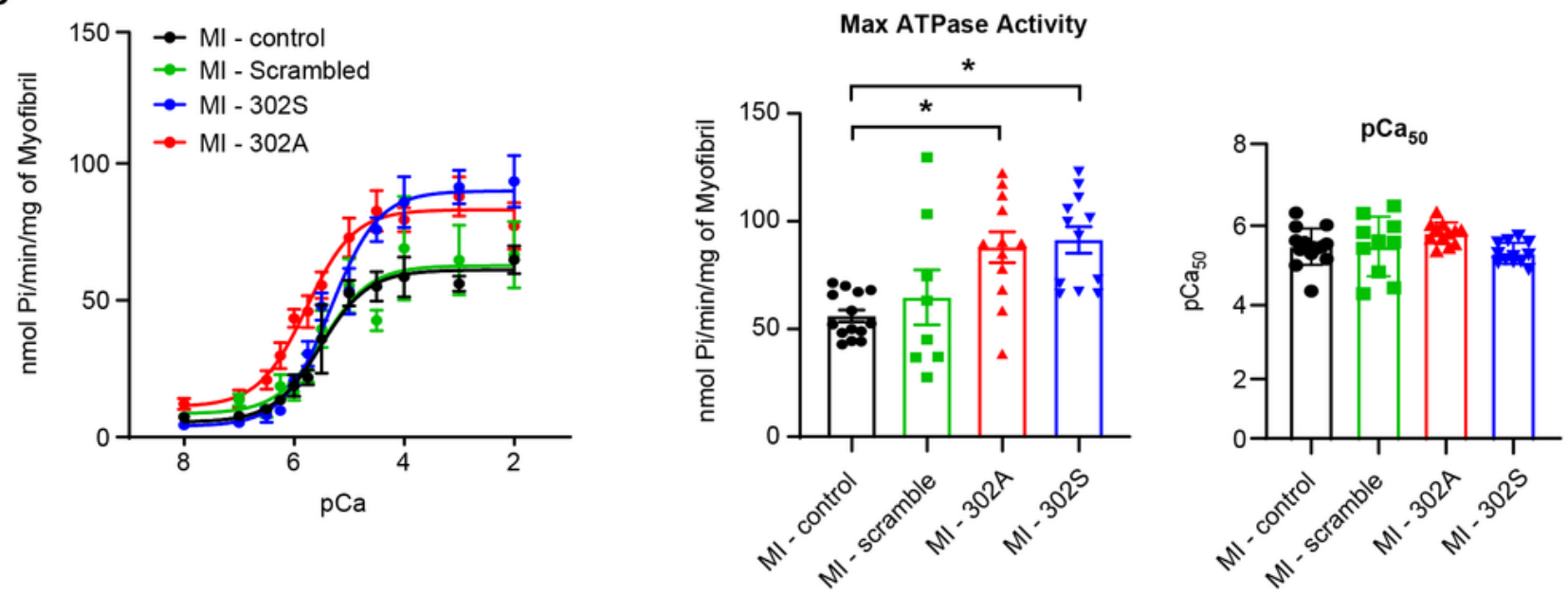

Figure 5

Myofibril ATPase activity was increased by cMyBP-C peptides 302A and 302S in week 1 post-MI rats but not in sham rats. (A) ATPase activity was reduced for more than $50 \%$ in myofibrils from the Ml infarct zone, but not changed in myofibrils from the Ml remote zone compared to myofibrils from sham hearts. 
Myofibril ATPase activity was normalized by myofibril protein used in the assay. (B) cMyBP-C peptides 302A and 302S did not change maximal ATPase activity in myofibrils isolated from sham rats. (C) cMyBP-C peptides 302A and 302S were able to increase maximal ATPase activity in myofibrils isolated from the infarct zone of week 1 post-MI rats compared to non-treatment control and to scrambled peptide-treated myofibrils. Data represent mean \pm SEM $(N=8-15) ; * p<0.05 ; * \star * p<0.005$; one-way ANOVA with Tukey post-test. MI, myocardial infarction.

A
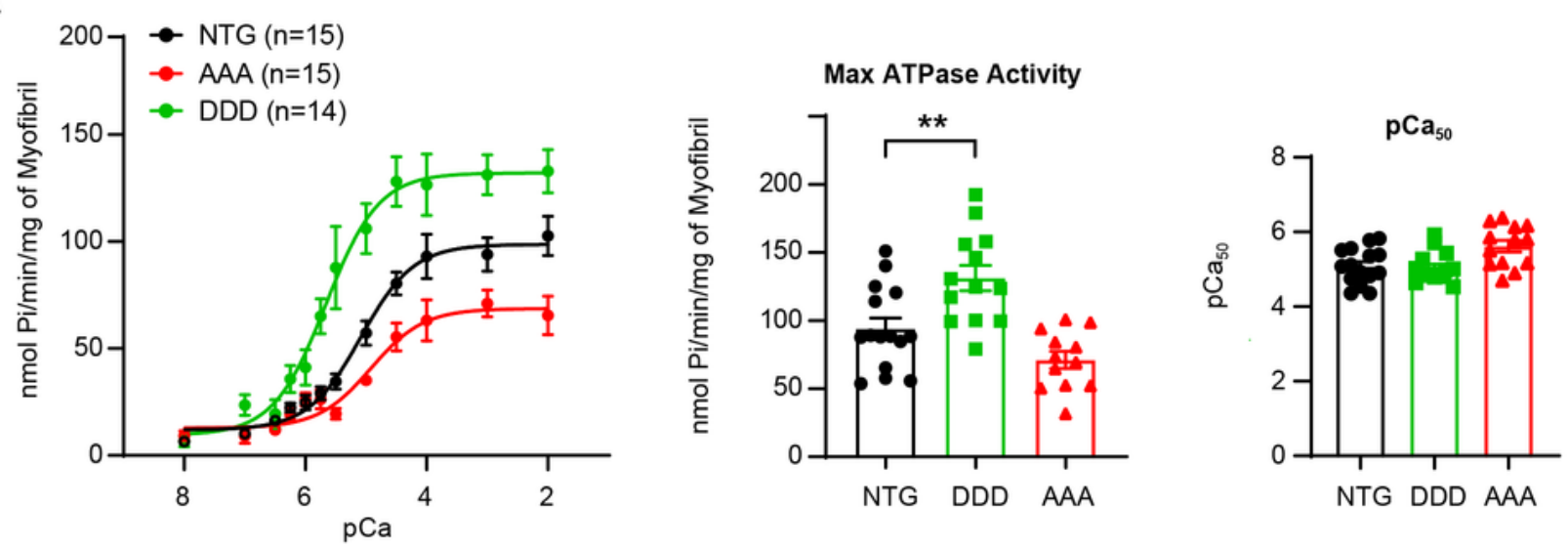

B
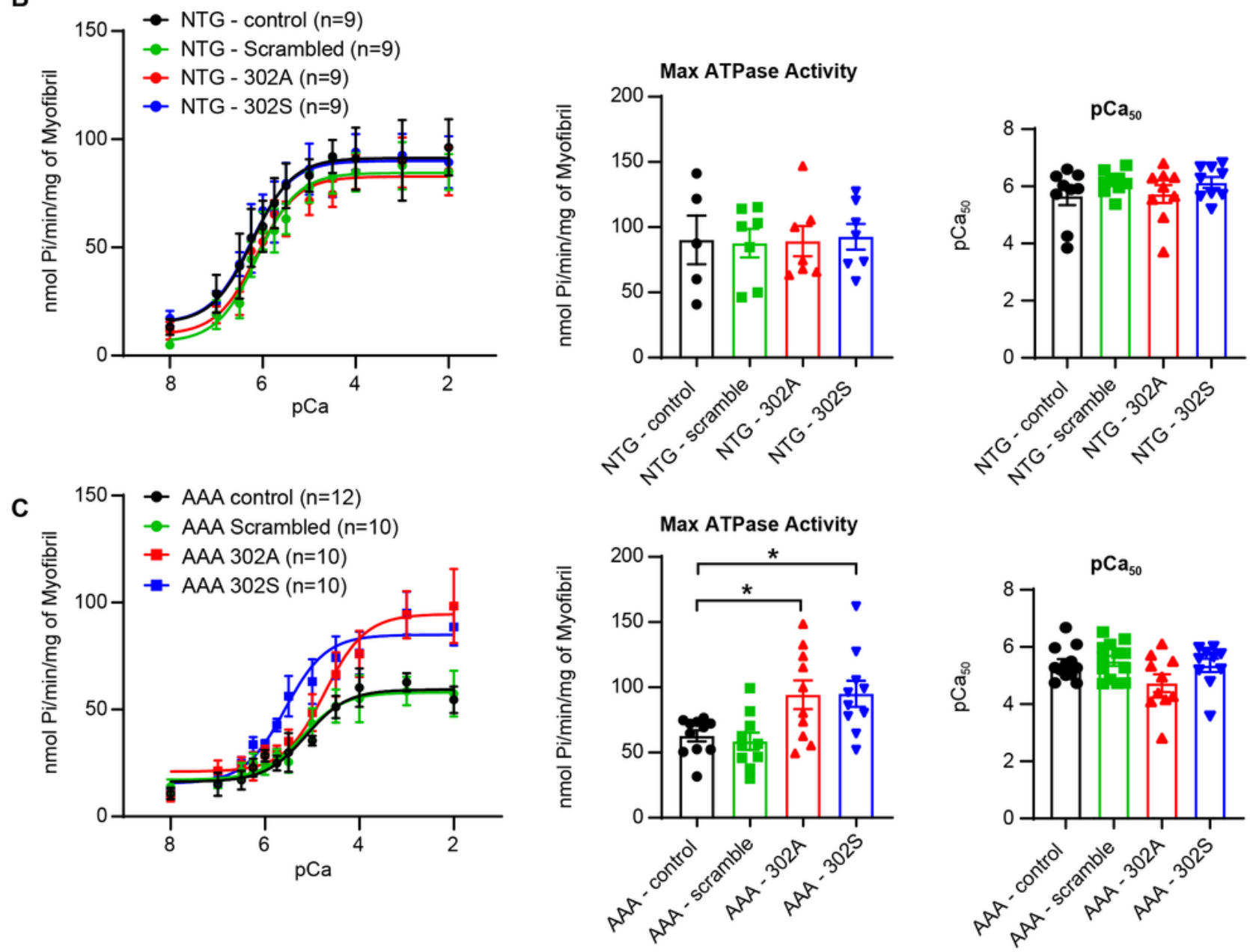

Figure 6 
Myofibril ATPase activity was increased by cMyBP-C peptides 302A and 302S in AAA TG mice, but not in NTG control mice. (A) ATPase activity was significantly increased in myofibrils isolated from DDD mice and were decreased in myofibrils from AAA mice compared to NTG mice. (B) cMyBP-C peptides 302A and $302 S$ did not have any effect on the maximal ATPase activity in myofibrils isolated from NTG mice. (C) cMyBP-C peptides 302A and 302S peptides were able to increase maximal ATPase activity in myofibrils isolated from AAA mice. ${ }^{*} \mathrm{p}<0.05$; ${ }^{*} \mathrm{p}<0.01$; one-way ANOVA with Tukey post-test. Data represent mean \pm SEM (N=9-15). AAA, nonphosphorylated alanines; DDD, phospho-mimetic aspartic acids; NTG, nontransgenic mice 


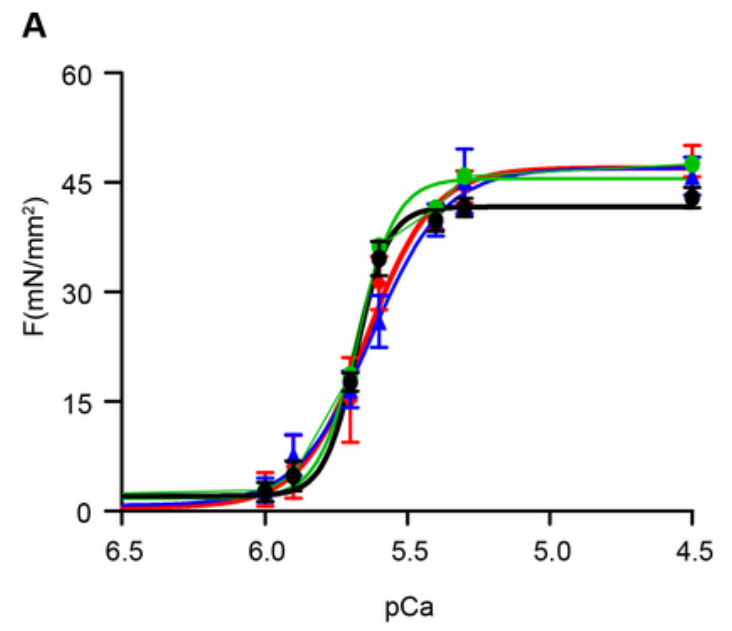

C

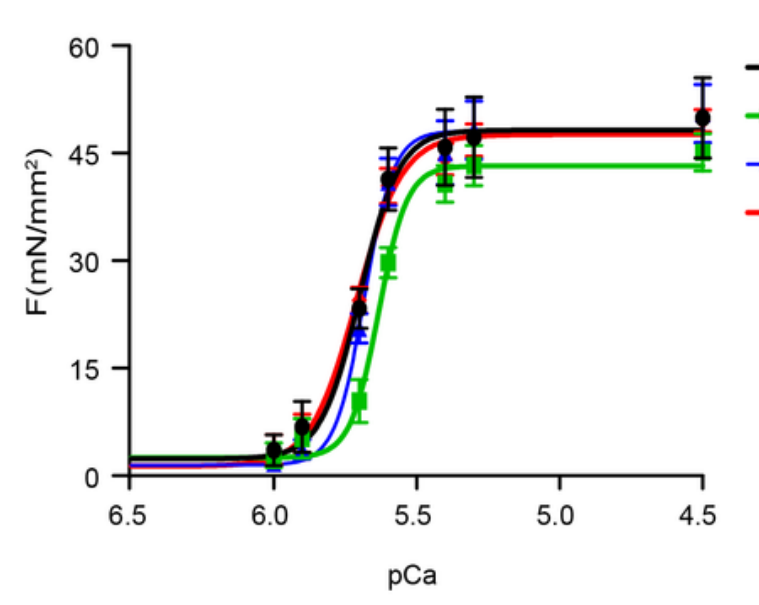

E

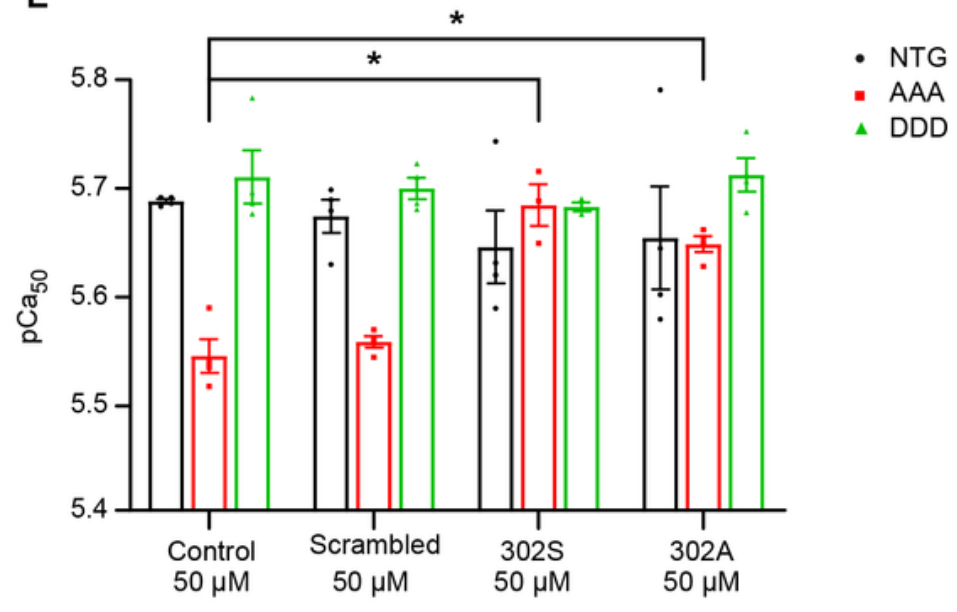

B

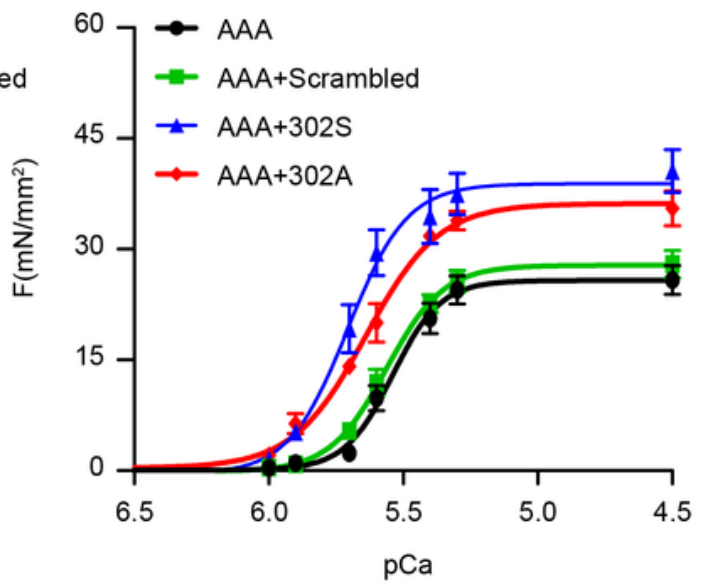

D
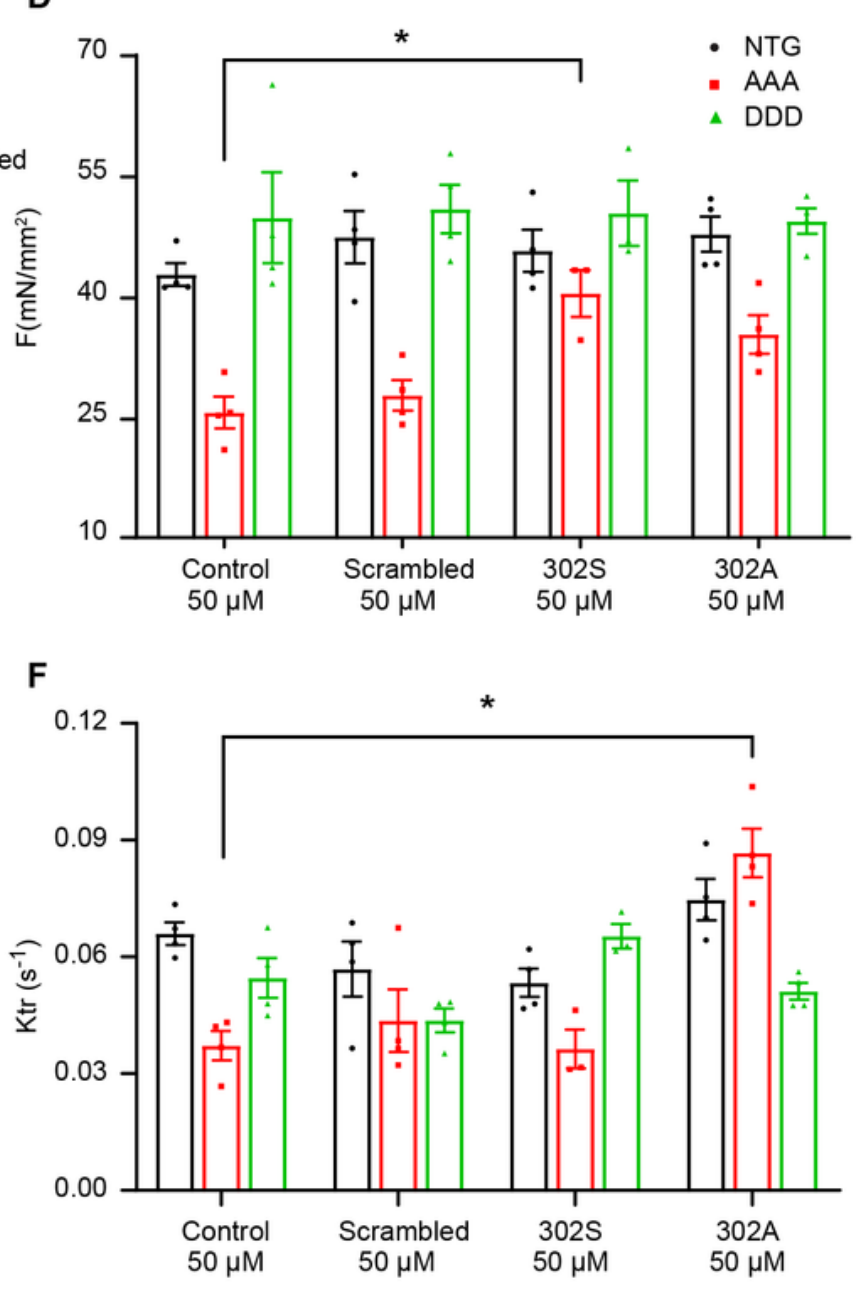

\section{Figure 7}

Cardiac function was improved by CMyBP-C peptide 302A and 302S in muscle fibers isolated from AAA transgenic mice, but not in NTG and DDD TG mice. Force pCa curves (A, B and C) generated at sarcomere length $2.0 \mu \mathrm{M}$ in the presence of different peptides $(50 \mu \mathrm{M})$. Maximum force production $(\mathrm{D}, \mathrm{mN} / \mathrm{mm} 2)$, calcium sensitivity (E, pCa50), and rate of force regeneration ( $F, k t r)$ were measured in the presence of different peptides $(50 \mu \mathrm{M})$ in NTG (white bar), AAA (red bar) and DDD (green bar). $\mathrm{n}=4$ papillary fibers 
per peptide treatment from 4 animals per group. ${ }^{*} p<0.05$ one-way ANOVA with Tukey post-test. AAA, nonphosphorylated alanines; DDD, phospho-mimetic aspartic acids; NTG, non-transgenic mice.
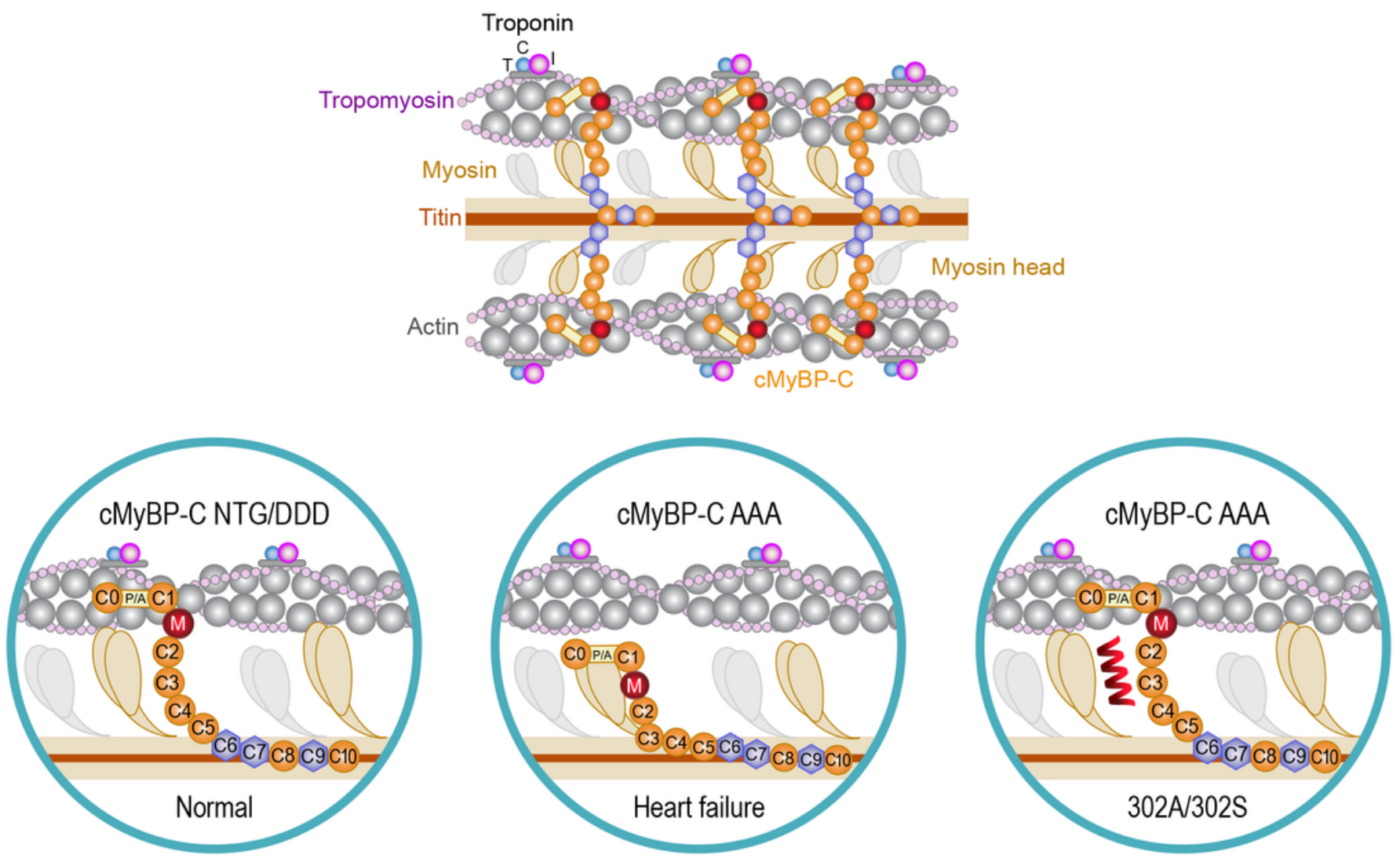

Figure 8

Diagram showing the effect of phosphorylation of cMyBP-C and the interaction of cMyBP-C peptide 302A and $302 \mathrm{~S}$ with sarcomere proteins. In the absence of phosphorylation (top), the position of the actinbinding site of CMyBP-C would lie about $3 \mathrm{~nm}$ from the thin filament. Phosphorylation of CMyBP-C (middle) would extend the cross-bridge to the surface of the thin filament and thereby loosen the packing of the rod portion of the myosin molecule. Permeabilizing 302A peptides inhibits myosin from interacting with cMyBP-C (bottom), resulting in its movement toward actin, which in turn results in the formation of actomyosin interactions and cross-bridges, thus enhancing contractility.

\section{Supplementary Files}

This is a list of supplementary files associated with this preprint. Click to download.

- 20210930SciRepSupFigurescMyBPCLH.pdfAT.pdf 